\title{
The Principal Eigenvalue Problems for Perturbed Fractional Laplace Operators
}

\author{
Guangyu Zhao
}

\begin{abstract}
We study a variety of basic properties of the principal eigenvalue of a perturbed fractional Laplace operator and weakly coupled cooperative systems involving fractional Laplace operators. Our work extends a number of well-known properties regarding the principal eigenvalues of linear second order elliptic operators with Dirichlet boundary condition to perturbed fractional Laplace operators. The establish results are also utilized to investigate the spatio-temporary dynamics of population models.
\end{abstract}

\section{Introduction}

In this paper, we are concerned with the principal eigenvalue problem for a perturbed fractional Laplace operator:

$$
\left\{\begin{array}{l}
(-\Delta)^{s} u+c(x) u=\lambda u \text { in } \Omega \\
u=0 \text { in } \mathbb{R}^{n} \backslash \Omega
\end{array}\right.
$$

Here $\Omega$ is a bounded domain of $\mathbb{R}^{n}$ with smooth boundary, $c \in L^{\infty}(\Omega)$, and

$$
(-\Delta)^{s} u=c_{n, s} \text { P.V. } \int_{\mathbb{R}^{n}} \frac{u(x)-u(y)}{|x-y|^{n+2 s}} d y:=c_{n, s} \lim _{\epsilon \rightarrow 0} \int_{|x-y|>\epsilon} \frac{u(x)-u(y)}{|x-y|^{n+2 s}} d y, \quad s \in(0,1),
$$

where $c_{n, s}=\frac{4^{s} s \Gamma\left(\frac{N}{2}+s\right)}{\pi^{\frac{n}{2}} \Gamma(1-s)}$ is the normalization constant. As shown in Proposition 4.4 of [20], $\lim _{s \rightarrow 1^{-}}(-\Delta)^{s} u=-\Delta u$ for any $u \in C_{0}^{\infty}\left(\mathbb{R}^{n}\right)$. The paper also addresses the principal eigenvalue problems for weakly coupled cooperative systems involving fractional Laplace operators as follows:

$$
\left\{\begin{array}{l}
(-\Delta)^{s_{i}} u_{i}+\sum_{j=1}^{k} c_{i, j}(x) u_{j}=\lambda u_{i} \quad \text { in } \Omega \quad(1 \leq i \leq k) \\
u_{i}=0 \text { in } \mathbb{R}^{n} \backslash \Omega,
\end{array}\right.
$$

where $s_{i} \in(0,1)$, and $c_{i, j} \in L^{\infty}(\Omega)$. Its main goal is to examine basic properties of the principal eigenvalues of the perturbed fractional Laplace operators given above, which would be similar to

Key words and phrases. Fractional Laplace operator, Principal eigenvalue, Maximum principle, weakly coupled cooperative systems.

2010 Mathematics Subject Classification. 35B36, 35J05, 35P15, 45K05. 
those for the principal eigenvalues of linear second order elliptic operators with Dirichlet boundary condition.

The principal eigenvalue problems of linear second order elliptic operators are fundamental to the theory and applications of partial differential equations and have been extensively studied over past few decades (see [1],[2],[11], and [17]). It is well known that the principal eigenvalues of linear second order elliptic operators play a crucial role in the study of spatio-temporary dynamics of population models. As shown in [8], just like the principal eigenvalues of other operators describing the dispersal and demographics of populations, the principal eigenvalues of linear second order elliptic operators can provide the intrinsic growth rates for spatially structured populations described by reaction-diffusion models. Meanwhile nonlocal diffusion equations have gained considerable popularity recently, due to their demonstrated applications in numerous diverse and widespread fields of science and engineering (see [3], [4], [6], [7], [15], [10], [21], [23], [24], [26] and references therein). In fact, perturbed fractional Laplace operators like the ones given in (1.1) and (1.2) often arise as a result of the linearization at an equilibrium of a semi-linear equation involving the fractional Laplacian. Therefore, under suitable boundary conditions, it is a natural question to ask if the principal eigenvalues of perturbed fractional Laplace operators still enjoy the essential features of the principal eigenvalues of linear second order elliptic operators.

In Dyda [12], a set of formulas are established for fractional calculus of power functions, and these formulas are employed to estimate upper and lower bounds of the principal eigenvalue of $(-\Delta)^{s}$ in a ball. Servadei and Valdinoci [23] investigates the eigenvalue problem of a non-local integro-differential operator with homogeneous Dirichlet boundary conditions, which along with [17] form the basis of our study. More recently, Massaccesi and Valdinoci [19] examined the distinction between local and non-local dispersal strategies in an attempt to analyze and to understand the impacts of different diffusive strategies on interacting species. Their work focus on a spatial model that describes the competition between two populations dispersing in different manners. More precisely, they assumed that the motion of one population is governed by a random walk while the other's movement is subject to nonlocal dispersal that follows a power law. A reverse Poincaré-Sobolev condition is given in [19], which serves as an indicator for the abundance of resources. Among other things, the condition was repeatedly used to study the existence and linear stability of an equilibrium of the model revealing the crucial role of the condition in determining the evolutionary stability of different dispersal strategies. It will become clear in this paper that the validity of the reverse Poincaré-Sobolev condition of [19] is equivalent to the negativity of the principal eigenvalue of (1.1).

Inspired by the above works and motivated by a characterization theorem in [17], which manifests the equivalence between the validity of a strong maximum principle and the positivity of the principal eigenvalue of linear second order elliptic operators under suitable conditions, the present work extends the characterization theorem and other basic properties regarding principal 
eigenvalue for linear second order elliptic operators to perturbed fractional Laplace operators with homogeneous Dirichlet boundary condition given in (1.1). The same characterization is also established for (1.2). This paper is organized as follows: in section 2, we investigate the equivalence between the validity of a strong maximum principle and the positivity of the principal eigenvalue of (1.1). As immediate consequences of this equivalence, it can be shown that the principal eigenvalue of (1.1) is monotone and continuous with respect to $c$, and it is also continuous with respect to a regular class of perturbations of $\Omega$ around its boundary.

We also study the existence and multiplicity of principal eigenvalues for a linear weighted eigenvalue problem. Section 3 is focused on the principal eigenvalue of (1.2). The aforementioned equivalence is obtained for (1.2) as well under certain conditions on $c_{i, j}$. This equivalence reveals the interplays between the sign of the principal eigenvalue of (1.2), the validity of the strong maximum principle, and the existence of a positive super-solution. As a result of the equivalence, the maximum principle for domains of small volume is also established for weakly coupled cooperative systems. Finally, in section 4 , the results established in the previous sections are utilized to study several prototype models that arise in mathematical ecology. More specifically, we study a nonlocal Ross-Macdonald model. It is shown that the principal eigenvalue plays a key role similar to the basic reproduction number when it comes to determining infection dynamics.

Throughout the paper, let $\Omega$ be an open and bounded subset of $\mathbb{R}^{n}$, we always write

$$
d(x, \partial \Omega)=\inf \left\{|x-y| \mid y \in \mathbb{R}^{n} \backslash \Omega\right\}
$$

for the distance of $x$ to the boundary $\partial \Omega$ of $\Omega$. Given $x \in \mathbb{R}^{n}, B_{r}(x):=\left\{y \in \mathbb{R}^{n}|| y-x \mid<r\right\}$. Given $u \in L^{\infty}(\Omega), u \geqslant 0$ in $\Omega$ means that $u \geq 0$ in $\Omega$, and there exists $x^{\prime} \in \Omega$ and $r>0$ for which $B_{r}\left(x^{\prime}\right) \subset \Omega$ and $\inf _{x \in B_{r}\left(x^{\prime}\right)} u>0 . u \lessgtr 0$ in $\Omega$ if $-u \gtrless 0$ in $\Omega$. $L_{\text {loc }}^{\infty}\left(\mathbb{R}^{n}\right)$ denotes the space of functions $u \in L^{\infty}(D)$ for any bounded measurable subset $D \subset \mathbb{R}^{n}$.

\section{The existence of principal eigenvalue and its properties}

In the sequel, we let $H_{0}^{s}(\Omega)$ be the Hilbert space defined as the closure of $C_{0}^{\infty}(\Omega)$ with respect to the norm

$$
\|u\|_{H_{0}^{s}(\Omega)}:=\left(\int_{\Omega}|u|^{2} d x\right)^{\frac{1}{2}}+\left(\int_{\mathbb{R}^{n}} \int_{\mathbb{R}^{n}} \frac{|u(x)-u(y)|^{2}}{|x-y|^{n+2 s}} d y d x\right)^{\frac{1}{2}} .
$$

Notice that $H_{0}^{s}(\Omega)$ is a subspace of $H^{s}\left(\mathbb{R}^{n}\right)$ consisting of functions that vanish outside $\Omega$. As shown in [24], $H_{0}^{s}(\Omega)$ is equipped with the following equivalent norm

$$
\|u\|_{H_{0}^{s}(\Omega)}:=\left(\int_{\Omega}|u|^{2} d x\right)^{\frac{1}{2}}+\left(\int_{\mathbb{R}^{2 n} \backslash \Omega^{c} \times \Omega^{c}} \frac{|u(x)-u(y)|^{2}}{|x-y|^{n+2 s}} d y d x\right)^{\frac{1}{2}},
$$

where $\mathbb{R}^{2 n}:=\mathbb{R}^{n} \times \mathbb{R}^{n}$ and $\Omega^{c}=\mathbb{R}^{n} \backslash \Omega$. 
Definition 1. Let $\Omega \subset \mathbb{R}^{n}$ be a bounded domain. Given $f \in L^{2}(\Omega)$, a function $w \in H_{0}^{s}(\Omega)$ is said to be a weak solution to the boundary value problem

$$
\left\{\begin{array}{l}
(-\Delta)^{s} u+c(x) u=f \quad \text { in } \Omega, \\
u=0 \quad \text { in } \mathbb{R}^{n} \backslash \Omega
\end{array}\right.
$$

provided that

$$
\frac{c_{n, s}}{2} \int_{\mathbb{R}^{n}} \int_{\mathbb{R}^{n}} \frac{[w(x)-w(y)][\phi(x)-\phi(y)]}{|x-y|^{n+2 s}} d y d x+\int_{\Omega} c w \phi d x=\int_{\Omega} f \phi d x
$$

for any $\phi \in H_{0}^{s}(\Omega)$.

Thereafter, for the sake of convenience, we shall write

$$
\left\{\begin{array}{l}
\left(L_{c} u\right)(x):=(-\Delta)^{s} u+c(x) u, \quad x \in \Omega, \\
\mathcal{D} u:=u, \quad x \in \mathbb{R}^{n} \backslash \Omega .
\end{array}\right.
$$

Let $\lambda \in \mathbb{R}, \lambda$ is said to be an eigenvalue of the eigenvalue problem (1.1), or equivalently, an eigenvalue of $\left\{L_{c}, \mathcal{D}, \Omega\right\}$ if there exists a function $u \in H_{0}^{s}(\Omega)$ such that $u$ is a weak solution of (2.1) with $f=\lambda u$. Depending on our needs, we also consider classical solutions of (2.1), following [21], assume that $f \in L^{\infty}(\Omega)$, a classical solution to (2.1) is a continuous function $u \in C\left(\mathbb{R}^{n}\right)$ such that $(-\Delta)^{s} u+c(x) u$ is well defined in $\Omega$ and $(-\Delta)^{s} u+c(x) u=f$ pointwise in $\Omega$.

Proposition 2.1. Let $\Omega \subset \mathbb{R}^{n}$ be a bounded domain with Lipschitz boundary. Suppose that $n>2 s$ and $w \in H_{0}^{s}(\Omega)$ is a weak solution to (2.1). Assume that $c, f \in L^{p}(\Omega)$ and $p>\max \{2, n / 2 s\}$. Then

$$
\|w\|_{L^{\infty}(\Omega)} \leq C
$$

for some positive constant $C$ depending only on $n, s, p, \Omega,\|c\|_{L^{p}(\Omega)}$, and $\|f\|_{L^{p}(\Omega)}$.

Proof. The Moser's iteration presented in [6] is utilized to prove the proposition. Given $m>0$, let $w_{m}$ be defined by $w_{m}=\min \{|w|, m\}$. Clearly, $w_{m} \in H_{0}^{s}(\Omega)$. In view of Lemma A.1 of [6], there holds

$$
\frac{c_{n, s}}{2} \int_{\mathbb{R}^{n}} \int_{\mathbb{R}^{n}} \frac{[|w(x)|-|w(y)|][\phi(x)-\phi(y)]}{|x-y|^{n+2 s}} d y d x \leq \int_{\Omega}|c||w| \phi d x+\int_{\Omega}|f| \phi d x
$$

for any positive $\phi \in H_{0}^{s}(\Omega)$. Now choose $\phi=\left(w_{m}+1\right)^{\beta}-1$, then following the line of Theorem 3.1 of [6] gives that

$$
\left(\int_{\Omega}\left(w_{m}+1\right)^{\frac{(1+\beta) n}{n-2 s}} d x\right)^{\frac{n-2 s}{n}} \leq C(n, s)\left(\frac{\beta+1}{2 \sqrt{\beta}}\right)^{2}\left\{K(c, f, \Omega)\left(\int_{\Omega}\left[(|w|+1)\left(w_{m}+\delta\right)^{\beta}\right]^{q} d x\right)^{\frac{1}{q}}\right\}
$$


where $q=\frac{p}{p-1}, K(c, f, \Omega)=\|c\|_{L^{p}(\Omega)}+\|f\|_{L^{p}(\Omega)}+|\Omega|^{\frac{n-2 s}{n}-\frac{1}{q}}$, and $C(n, s)>0$ is constant depending only on $n$ and $s$. Now let $m \rightarrow \infty$, thanks to the monotone convergence theorem, by passing the limits in the above inequality, we find that

$$
\left(\int_{\Omega}(|w|+1)^{\frac{(1+\beta) n}{n-2 s}} d x\right)^{\frac{n-2 s}{n}} \leq C(n, s)\left(\frac{\beta+1}{2 \sqrt{\beta}}\right)^{2}\left\{K(c, f, \Omega)\left(\int_{\Omega}\left[(|w|+1)^{(1+\beta) q}\right] d x\right)^{\frac{1}{q}}\right\} .
$$

Let $\theta=(1+\beta) q$ and $\chi=\frac{n}{(n-2 s) q}$, note that there exists a constant $\gamma_{q}$, depending on $q$ only, such that $\frac{\beta+1}{2 \sqrt{\beta}} \leq \sqrt{\gamma_{q}(\beta+1)}$ as long as $\beta \geq \frac{2-q}{q}$. If $\beta \geq \frac{2-q}{q}$, write $C(n, s, q)=C(n, s) \gamma_{q}$, then

$$
\left(\int_{\Omega}(|w|+1)^{\chi \theta} d x\right)^{\frac{1}{\chi^{\theta}}} \leq[K(c, f, \Omega)]^{\frac{q}{\theta}}\left(\frac{C(n, s, q) \theta}{q}\right)^{\frac{q}{\theta}}\left(\int_{\Omega}(|w|+1)^{\theta} d x\right)^{\frac{1}{\theta}} .
$$

It follows from the assumption that $\chi>1$. Now choose $\theta_{0}=2$, and let $\theta_{n+1}=\chi \theta_{n}$ with $n \in \mathbb{N}^{+}$, after $m$ iterations, we have

$$
\|(|w|+1)\|_{L^{\theta_{m+1}(\Omega)}} \leq[K(c, f, \Omega)]^{\sum_{i=0}^{m} \frac{q}{\theta_{i}}} \prod_{i=0}^{m}\left(\frac{C(n, s, q) \theta_{i}}{q}\right)^{\frac{q}{\theta_{i}}}\|(|w|+1)\|_{L^{2}(\Omega)} .
$$

By passing the limit as $m \rightarrow \infty$, the desired conclusion follows. The proof is completed.

Proposition 2.2. Let $\Omega \subset \mathbb{R}^{n}$ be a bounded domain with Lipschitz boundary. Let $L_{c}$ and $\mathcal{D}$ be given by (2.2). Then $\left\{L_{c}, \mathcal{D}, \Omega\right\}$ has a countable family of eigenvalues $\left\{\lambda_{k}\right\}_{k=1}^{\infty}$ that can be written as an increasing sequence approaching $+\infty$ as $k \rightarrow \infty$. Namely,

$$
-\infty<\lambda_{1}<\lambda_{2} \leq \lambda_{3} \leq \cdots \leq \lambda_{k} \leq \cdots
$$

In addition, there holds

$$
\begin{gathered}
\lambda_{1}=\inf _{u \in H_{0}^{s}(\Omega),\|u\|_{L^{2}(\Omega)}=1} \frac{c_{n, s}}{2} \int_{\mathbb{R}^{n}} \int_{\mathbb{R}^{n}} \frac{|u(x)-u(y)|^{2}}{|x-y|^{n+2 s}} d y d x+\int_{\Omega} c(x) u^{2} d x, \\
\lambda_{k}=\inf _{u \in X_{k-1}^{\perp},\|u\|_{L^{2}(\Omega)}=1} \frac{c_{n, s}}{2} \int_{\mathbb{R}^{n}} \int_{\mathbb{R}^{n}} \frac{|u(x)-u(y)|^{2}}{|x-y|^{n+2 s}} d y d x+\int_{\Omega} c(x) u^{2} d x, \quad k \geq 2,
\end{gathered}
$$

where $X_{k}=\bigoplus_{i=1}^{k} \operatorname{ker}\left(L_{c}-\lambda_{i} I\right)$. Each $\lambda_{k}$ has finite geometric multiplicity. Moreover, the eigenfunctions associated with $\left\{\lambda_{k}\right\}_{k=1}^{\infty}$ form an orthonormal basis of $H_{0}^{s}(\Omega)$. Assume further that $\Omega$ satisfies the exterior ball condition, and $\varphi_{k} \in X_{k}$. Then $\varphi_{k} \in C^{s}\left(\mathbb{R}^{n}\right)$, and

$$
L_{c} \varphi_{k}=\lambda_{k} \varphi_{k} \quad \text { in } \Omega, \quad \varphi_{k}=0 \quad \text { in } \mathbb{R}^{n} \backslash \Omega
$$


Proof. The proof of the existence of $\left\{\lambda_{k}\right\}_{k=1}^{\infty}$ is the same as that in [23], while $\lambda_{1}$ is referred to as the principal eigenvalue of $\left\{L_{c}, \mathcal{D}, \Omega\right\}$, which is a simple eigenvalue. Let $\varphi_{i}$ be an eigenvalue associated with $\lambda_{i}$. Owing to Proposition 2.1, $\varphi_{i} \in L^{\infty}(\Omega)$. In particular, similar to [23], we can show that $\varphi_{1}(x)>0\left(\right.$ or $\varphi_{1}(x)<0$ ) for all $x \in \Omega$. In case that $\Omega$ is a Lipschitz domain satisfying the exterior ball condition, then it follows from [21] that $\varphi_{i} \in C^{s}\left(\mathbb{R}^{n}\right)$ satisfies $L_{c} \varphi_{i}=\lambda_{i} \varphi_{i}$ point-wise in $\Omega$. The proof is completed.

Let $\lambda_{1}$ be given by (2.3). In what follows, we denote $\lambda_{1}$ by $\lambda_{s}$, and $\lambda_{s}$ is referred to as the principle eigenvalue of $\left\{L_{c}, \mathcal{D}, \Omega\right\}$. Namely, $\lambda_{s}$ is the least eigenvalue of the eigenvalue problem (1.1), and it is the only eigenvalue of $\left\{L_{c}, \mathcal{D}, \Omega\right\}$ with positive eigenfunctions.

Definition 2. [17] Let $\Omega \subset \mathbb{R}^{n}$ be a domain. $\Omega$ is said to satisfy the interior sphere property at a single point $x \in \partial \Omega$ if there exist $z_{x} \in \Omega$ and $r>0$ such that $\left|x-z_{x}\right|=r$ and $B_{r}\left(z_{x}\right) \subset \Omega$.

Definition 3. [17] Let $\Omega \subset \mathbb{R}^{n}$ be a domain. $\Omega$ is said to satisfy the uniform interior sphere property in the strong sense if there exists $r>0$ such that for every $x \in \Omega$ with $d(x, \partial \Omega) \leq r$, there exists a point $z_{x} \in \partial \Omega$ for which $\left|x-z_{x}\right|=d(x, \partial \Omega)$ and $B_{r}\left(z_{x}+r \frac{x-z_{x}}{\left|x-z_{x}\right|}\right) \subset \Omega$.

Definition 4. Let $u \in L_{\text {loc }}^{\infty}\left(\mathbb{R}^{n}\right) \cap C(\bar{\Omega}), u$ is said to be a (classical) super-solution of $\left\{L_{c}, \mathcal{D}, \Omega\right\}$ if $L_{c} u \in L^{\infty}(\Omega)$, and

$$
(-\Delta)^{s} u+c(x) u \geq 0 \text { in } \Omega, \quad \text { and } u \geq 0 \text { in } \mathbb{R}^{n} \backslash \Omega
$$

In addition, $u$ is said to be a strict super-solution of $\left\{L_{c}, \mathcal{D}, \Omega\right\}$ provided that $u$ is a (classical) super-solution of $\left\{L_{c}, \mathcal{D}, \Omega\right\}$ while $u$ is not classical solution of $L_{c} u=0$ in $\Omega$ and $u=0$ in $\mathbb{R}^{n} \backslash \Omega$.

Proposition 2.3. [15] Let $u \in L_{\text {loc }}^{\infty}\left(\mathbb{R}^{n}\right) \cap C(\bar{\Omega})$ be a super-solution of $\left\{L_{c}, \mathcal{D}, \Omega\right\}$. Assume that $c \in L^{\infty}(\Omega)$ and $c \geq 0$. Then either $u=0$ in $\mathbb{R}^{n}$ or $u>0$ in $\Omega$.

Proof. The proof can be found in [15](see the proof for Theorem 2.1 of [15]).

Lemma 2.1. Let $\Omega \subset \mathbb{R}^{n}$ be a domain (either a bounded or an unbounded domain). Suppose that $\Omega$ satisfies the interior sphere property at a point $x_{0} \in \partial \Omega$. Let $z_{x_{0}} \in \Omega$ such that $\left|x_{0}-z_{x_{0}}\right|=r$ and $B_{r}\left(z_{x_{0}}\right) \subset \Omega$. Assume that $u \in C\left(\bar{B}_{r}\left(z_{x_{0}}\right)\right) \cap L_{\text {loc }}^{\infty}\left(\mathbb{R}^{n}\right)$ is a super-solution of $\left\{L_{c}, \mathcal{D}, B_{r}\left(z_{x_{0}}\right)\right\}$ and $u \geqslant 0$ in $\mathbb{R}^{n}$. Then there exist positive constants $\rho$ and $r_{1}$ with $0<r_{1}<r$ such that $u(x) \geq$ $\theta \rho d\left(x, \partial B_{r}\left(z_{x_{0}}\right)\right)^{s}$ for all $x \in B_{r}\left(z_{x_{0}}\right)$, where $\theta=\inf _{x \in \bar{B}_{r_{1}}\left(z_{x_{0}}\right)} u$, and $r_{1}$, $\rho$ depend on $r, s, n$ and $\left|c_{+}\right|_{L^{\infty}(\Omega)}$ only. 
Proof. Set

$$
w_{1}(x)=\left(r^{2}-\left|x-z_{x_{0}}\right|^{2}\right)_{+}^{s}, \quad w_{2}(x)=\left(r^{2}-\left|x-z_{x_{0}}\right|^{2}\right)_{+}^{1+s}, \quad x \in \mathbb{R}^{n} .
$$

It then follows from Table 3 of [12] that

$$
\begin{aligned}
(-\Delta)^{s} w_{1}(x)=2^{2 s} \Gamma(1+s) \Gamma\left(\frac{n}{2}+s\right) \Gamma\left(\frac{n}{2}\right)^{-1}=k_{1}, \quad x \in B_{r}\left(z_{x_{0}}\right), \\
(-\Delta)^{s} w_{2}(x)=2^{2 s} \Gamma(2+s) \Gamma\left(\frac{n}{2}+s\right) \Gamma\left(\frac{n}{2}\right)^{-1}\left(r^{2}-\left(1+\frac{2 s}{n}\right)\left|x-z_{x_{0}}\right|^{2}\right), \quad x \in B_{r}\left(z_{x_{0}}\right) \\
=k_{2}\left(r^{2}-\left(1+\frac{2 s}{n}\right)\left|x-z_{x_{0}}\right|^{2}\right) .
\end{aligned}
$$

Since

$$
\lim _{\left|x-z_{x_{0}}\right| \rightarrow r}\left(r^{2}-\left(1+\frac{2 s}{n}\right)\left|x-z_{x_{0}}\right|^{2}\right)=-\frac{2 s}{n} r^{2}, \quad \lim _{\left|x-z_{x_{0}}\right| \rightarrow r} w_{1}=\lim _{\left|x-z_{x_{0}}\right| \rightarrow r} w_{2}=0,
$$

there exists $r_{1} \in(0, r)$ such that $k_{2}\left(r^{2}-\left(1+\frac{2 s}{n}\right)\left|x-z_{x_{0}}\right|^{2}\right)+\left|c_{+}\right|_{L_{\infty}}\left(w_{1}+w_{2}\right) \leq-\frac{s k_{2} r^{2}}{n}$ for all $x \in B_{r}\left(z_{x_{0}}\right) \backslash \bar{B}_{r_{1}}\left(z_{x_{0}}\right)$. Apparently, $r_{1}$ is determined by $r, s, n$, and $\left|c_{+}\right| L_{\infty}$ only, and is independent of $z_{x_{0}}$. Note that $\frac{s k_{2} r^{2}}{n k_{1}}=\frac{s \Gamma(1+s) r^{2}}{n}$. Let $k_{3}=\min \left\{1, \frac{s \Gamma(1+s) r^{2}}{n}\right\}$, it is easy to see that

$$
(-\Delta)^{s}\left[k_{3} w_{1}+w_{2}\right]+c_{+}(x)\left[k_{3} w_{1}+w_{2}\right] \leq 0 \quad \text { in } B_{r}\left(z_{x_{0}}\right) \backslash \bar{B}_{r_{1}}\left(z_{x_{0}}\right) .
$$

Now let $\theta=\inf _{x \in \bar{B}_{r_{1}}\left(z_{x_{0}}\right)} u$. Since $u \geqslant 0$ in $\mathbb{R}^{n}$, and $(-\Delta)^{s} u+c_{+} u \geq 0$ for all $x \in B_{r}\left(z_{x_{0}}\right)$, it follows from Proposition 2.3 that $u>0$ in $B_{r}\left(z_{x_{0}}\right)$. Moreover, as $\bar{B}_{r_{1}}\left(z_{x_{0}}\right)$ is compact, we have $\theta>0$. Write $v=\frac{\theta}{r^{2 s}+r^{2+2 s}}\left(k_{3} w_{1}+w_{2}\right)$. Clearly, $\sup _{x \in \Omega} v \leq \theta$, it then follows that $u \geq v$ for all $x \in \bar{B}_{r_{1}}\left(z_{x_{0}}\right)$. In addition, the assumption shows that $u \geq v=0$ for all $x \in \mathbb{R}^{n} \backslash B_{r}\left(z_{x_{0}}\right)$. Meanwhile, there holds that $(-\Delta)^{s}(u-v)+c_{+}(u-v) \geq 0$ in $B_{r}\left(z_{x_{0}}\right) \backslash \bar{B}_{r_{1}}\left(z_{x_{0}}\right)$. Thus Proposition 2.3 implies that $u>v$ for all $x \in B_{r}\left(z_{x_{0}}\right) \backslash \bar{B}_{r_{1}}\left(z_{x_{0}}\right)$. Notice that

$$
v \geq \frac{\theta}{r^{2 s}+r^{2+2 s}}\left(k_{3} w_{1}\right) \geq \frac{r^{s} k_{3} \theta}{r^{2 s}+r^{2+2 s}}\left(r-\left|x-z_{x_{0}}\right|\right)_{+}^{s} .
$$

Let $\rho=\frac{r^{s} k_{3}}{r^{2 s}+r^{2+2 s}}$, we readily conclude that $u(x) \geq \theta \rho d\left(x, \partial B_{r}\left(z_{x_{0}}\right)\right)^{s}$ for all $x \in B_{r}\left(z_{x_{0}}\right) \backslash$ $\bar{B}_{r_{1}}\left(z_{x_{0}}\right)$. In particular, as a result of the fact that $u \geq v$ for all $x \in \bar{B}_{r_{1}}\left(z_{x_{0}}\right)$, there hold that $u \geq v$ for all $x \in B_{r}\left(z_{x_{0}}\right)$. In other words, $u \geq \theta \rho d\left(x, \partial B_{r}\left(z_{x_{0}}\right)\right)^{s}$ for all $x \in B_{r}\left(z_{x_{0}}\right)$. The proof is completed.

We now give a uniform decay property of E. Hopf for super-solutions of $\left\{L_{c}, \mathcal{D}, \Omega\right\}$, which will be used in serval places of this paper. 
Lemma 2.2. Assume that $\Omega \subset \mathbb{R}^{n}$ is a bounded domain satisfying the uniform interior sphere property in the strong sense. Let $u \in L_{\text {loc }}^{\infty}\left(\mathbb{R}^{n}\right) \cap C(\bar{\Omega})$ be a nonnegative super-solution of $\left\{L_{c}, \mathcal{D}, \Omega\right\}$, and $u \gtrless 0$ in $\mathbb{R}^{n}$. Then there exists $\beta>0$ such that $u(x) \geq \beta d(x, \partial \Omega)^{s}$ for all $x \in \Omega$. Assume further that $\partial \Omega$ is of class $\mathcal{C}^{1}$, and $u=0$ in $\mathbb{R}^{n} \backslash \Omega$. Then $\lim _{d(x, \partial \Omega) \rightarrow 0} \frac{u(x)}{d(x, \partial \Omega)^{s}} \geq \beta$.

Proof. Let $r>0$ be the constant specified in Definition 3. Given $x \in \Omega$ with $d(x, \partial \Omega) \leq r$, let $z_{x} \in \partial \Omega$ such that $d(x, \partial \Omega)=\left|x-z_{x}\right|$, we write $x^{\prime}=z_{x}+r \frac{x-z_{x}}{\left|x-z_{x}\right|}$. Due to the assumption, $B_{r}\left(x^{\prime}\right) \subset \Omega$. Clearly, $z_{x} \in \bar{B}_{r}\left(x^{\prime}\right) \cap \partial \Omega$. Let $r_{1} \in(0, r)$ be the constant found in the proof of Lemma 2.1 for $B_{r}\left(x^{\prime}\right)$. Again, $r_{1}$ depends on $r, n, s$ and $\left|c_{+}\right|_{L^{\infty}(\Omega)}$ only. Let $\Theta:=\{x \in \Omega \mid$ $\left.d(x, \partial \Omega) \geq \frac{r-r_{1}}{2}\right\}$. Notice that $d\left(x^{\prime}, \partial \Omega\right)=\left|x^{\prime}-z_{x}\right|=r$. It hence follows that $\bar{B}_{r_{1}}\left(x^{\prime}\right) \subset \Theta$. In fact, if there were a $y \in \bar{B}_{r_{1}}\left(x^{\prime}\right)$ and $y$ were not in $\Theta$, that is, $d(y, \partial \Omega)<\frac{r-r_{1}}{2}$. Let $z_{y} \in \partial \Omega$ such that $d(y, \partial \Omega)=\left|y-z_{y}\right|$. Then we would have $\left|x^{\prime}-z_{y}\right| \leq\left|x^{\prime}-y\right|+\left|y-z_{y}\right| \leq r_{1}+\frac{r-r_{1}}{2}=$ $\frac{r+r_{1}}{2}<r$, which is obviously a contradiction as $d\left(x^{\prime}, \partial \Omega\right)=r$. The contradiction confirms that $\bar{B}_{r_{1}}\left(x^{\prime}\right) \subset \Theta$. Slightly abusing the notation, we let $\theta=\inf _{x \in \Theta} u$. Then it follows from Lemma 2.1 that $u(y) \geq \theta \rho d\left(y, \partial B_{r}\left(x^{\prime}\right)\right)^{s}$ for $y \in B_{r}\left(x^{\prime}\right)$. Namely, $u(x) \geq \theta \rho d(x, \partial \Omega)^{s}$. Note that $x \in \Omega$ with $d(x, \partial \Omega) \leq r$ is arbitrary, and $\rho$ depends on $r, s, n$ and $\left|c_{+}\right|_{L^{\infty}(\Omega)}$ only. It is easy to see that $u(x) \geq \theta \rho d(x, \partial \Omega)^{s}$ for all $x \in \Omega$ with $d(x, \partial \Omega) \leq r$. Now let $\Theta^{\prime}=\{x \in \Omega \mid d(x, \partial \Omega) \geq r\}$. Clearly, $\Theta^{\prime}$ is a compact subset of $\Omega$. Let $\vartheta=\inf _{x \in \Theta^{\prime}} u$, then $\vartheta>0$ as $u>0$ in $\Omega$. Since $d(x, \partial \Omega)$ is bounded, there exists $\gamma>0$ such that $u \geq \gamma d(x, \partial \Omega)^{s}$ for all $x \in \Theta^{\prime}$. Set $\beta=\min \{\theta \rho, \gamma\}$, we readily conclude that $u(x) \geq d(x, \partial \Omega)^{s}$ for all $x \in \Omega$. Assume further that $\partial \Omega$ is of class $\mathcal{C}^{1}$, and $u=0$ in $\mathbb{R}^{n} \backslash \Omega$, Then it follows from [21] that $\frac{u}{d(x, \partial \Omega)^{s}} \in C^{\alpha}(\bar{\Omega})$ for some $\alpha \in(0, s)$. Hence $\lim _{d(x, \partial \Omega) \rightarrow 0} \frac{u(x)}{d(x, \partial \Omega)^{s}} \geq \beta$. The proof is completed.

Remark 1. Under the assumptions that are slightly weaker than those of Lemma 2.1, by deriving a contradiction, it is established in [15] that $\lim _{\inf }(x, \partial \Omega) \rightarrow 0 \frac{u(x)}{d(x, \partial \Omega)^{s}}>0$. While Lemma 2.1 presents a bit more explicit lower bound for $\frac{u(x)}{d(x, \partial \Omega)^{s}}$. In case that $\partial \Omega$ is sufficiently smooth, then the Hopf's lemma given in [15] can be restated as $\lim _{\epsilon \rightarrow 0} \frac{u(x-\epsilon \nu)}{\epsilon^{s}} \geq \beta$.

Definition 5. Let $L_{c}$ and $\mathcal{D}$ be defined in (2.2). Then $\left\{L_{c}, \mathcal{D}, \Omega\right\}$ is said to satisfy the strong maximum principle if $u>0$ in $\Omega$ whenever $u \in C^{s}(\bar{\Omega}) \cap L_{\mathrm{loc}}^{\infty}\left(\mathbb{R}^{n}\right)$ is a nontrivial super-solution of $\left\{L_{c}, \mathcal{D}, \Omega\right\}$.

Theorem 2.1. Assume that $\Omega \subset \mathbb{R}^{n}$ is a bounded domain with $\partial \Omega \in \mathcal{C}^{1}$. Let $\lambda_{s}$ be the principal eigenvalue of $\left\{L_{c}, \mathcal{D}, \Omega\right\}$. Then the following statements are equivalent

(i) $\lambda_{s}>0$,

(ii) $\left\{L_{c}, \mathcal{D}, \Omega\right\}$ has a positive strict super-solution $u \in C(\bar{\Omega}) \cap L_{\text {loc }}^{\infty}\left(\mathbb{R}^{n}\right)$,

(iii) $\left\{L_{c}, \mathcal{D}, \Omega\right\}$ satisfies the strong maximum principle; 
(iv) the linear boundary value problem

$$
\left\{\begin{array}{l}
(-\Delta)^{s} u+c(x) u=f \quad \text { in } \Omega, \\
u=0 \quad \text { in } \mathbb{R}^{n} \backslash \Omega
\end{array}\right.
$$

has an unique solution $u \in H_{0}^{s}(\Omega)$ for each $f \in L^{2}(\Omega)$. In particular, $u \geq 0$ provided that $f \geq 0$.

Proof. (i) implies (ii). This is obvious as a positive eigenfunction associated with $\lambda_{s}$ is a strict super-solution of $\left\{L_{c}, \mathcal{D}, \Omega\right\}$. (ii) implies (iii). Let $u \in C(\bar{\Omega}) \cap L_{\text {loc }}^{\infty}\left(\mathbb{R}^{n}\right)$ be the positive strict super-solution of $\left\{L_{c}, \mathcal{D}, \Omega\right\}$. Let $v \in C^{s}(\bar{\Omega}) \cap L_{\text {loc }}^{\infty}\left(\mathbb{R}^{n}\right)$ be a super-solution of $\left\{L_{c}, \mathcal{D}, \Omega\right\}$. We shall follow the idea of [1] to show that $v>0$ in $\Omega$. Let $v\left(x_{0}\right)=\inf _{\Omega} v(x)$ for some $x_{0} \in \bar{\Omega}$. It is sufficient to show that $v\left(x_{0}\right) \geq 0$. Assume to the contrary that this is not true, that is $v\left(x_{0}\right)<0$, then as $v \geq 0$ in $\Omega^{c}$, we have $x_{0} \in \Omega$. Given $x \in \Omega$. Let $\bar{x} \in \partial \Omega$ be the point such that $|x-\bar{x}|=d(x, \partial \Omega)$. Since $v \in C^{s}(\bar{\Omega})$, then there is $\kappa>0$ for which $v(x) \geq v(\bar{x})-\kappa d(x, \partial \Omega)^{s} \geq$ $-\kappa d(x, \partial \Omega)^{s}$. Consequently, $v(x) \geq-\kappa d(x, \partial \Omega)^{s}$ for all $x \in \bar{\Omega}$. Meanwhile, Lemma 2.2 shows that $u(x) \geq \beta d(x, \partial \Omega)^{s}$ for all $x \in \bar{\Omega}$ and some $\beta>0$. Hence there is $t^{\prime}>0$ such that $t^{\prime} u(x)+v(x) \geq 0$ for all $x \in \bar{\Omega}$. Now let $\bar{t}=\inf \{t \geq 0 \mid t u(x)+v(x) \geq 0$ in $\bar{\Omega}\}$. Clearly, $\bar{t}>0$ and $\bar{t} u(x)+v(x) \geq 0$ in $\bar{\Omega}$. Since $\bar{t} u(x)+v(x)$ is a strict super-solution of $\left\{L_{c}, \mathcal{D}, \Omega\right\}$, we have $\bar{t} u(x)+v(x)>0$ in $\Omega$. Thus, Lemma 2.2 shows that $\bar{t} u(x)+v(x) \geq \theta d(x, \partial \Omega)^{s}$ for all $x \in \bar{\Omega}$ and some $\theta>0$. Let $\vartheta=\frac{\theta}{2 \kappa}$. Then $\bar{t} u(x)+v(x) \geq-\vartheta v(x)$ for all $x \in \bar{\Omega}$. Hence $\frac{\bar{t}}{1+\vartheta} u(x)+v(x) \geq 0$ for all $x \in \bar{\Omega}$, which contradicts the definition of $\bar{t}$. Therefore, $v\left(x_{0}\right) \geq 0$. Particularly, Proposition 2.3 implies that $v(x)>0$ in $\Omega$. It is apparent that (iii) yields that (ii)

Next we show that (ii) implies (i). Assume to the contrary that $\lambda_{s} \leq 0$. Let $w$ be the strict super-solution and $\varphi$ be a positive eigenfunction. It follows from Proposition 1.1 of [21] that $|\varphi(x)| \leq C d(x, \partial \Omega)^{s}$. As $w \geq 0$ and $\varphi=0$ in $\mathbb{R}^{n} \backslash \Omega$, in particular, Lemma 2.2 shows that there exists $\gamma>0$ for which $w \geq \gamma d(x, \partial \Omega)^{s}$ for all $x \in \Omega$. Thus, there exists $t>0$ such that $t w-\varphi \geq 0$ for all $x \in \mathbb{R}^{n}$. Again let $\bar{t}=\{t \in \mathbb{R} \mid t w-\varphi \geq 0\}$. Apparently, $\bar{t}>0$. Note that $t w-\varphi$ is a super-solution of $L_{c}-\lambda_{s} I$. In addition, one of the followings must hold: $L_{c}(t w-\varphi)-\lambda_{s}(t w-\varphi) \gtrless 0$ in $\Omega$ or $t w-\varphi \supsetneqq 0$ in $\Omega^{c}$. With the same reasoning as above, we can reach a contradiction. Hence $\lambda_{s}>0$.

We now show that (i) implies (iv). Define

$$
I(u)=\frac{c_{n, s}}{4} \int_{\mathbb{R}^{n}} \int_{\mathbb{R}^{n}} \frac{|u(x)-u(y)|^{2}}{|x-y|^{n+2 s}} d y d x+\frac{1}{2} \int_{\Omega} c u^{2} d x-\int_{\Omega} f u d x .
$$

As

$$
0<\lambda_{s}=\inf _{u \in H_{0}^{s}\left(\mathbb{R}^{n}\right),\|u\|_{L^{2}}=1} \frac{c_{n, s}}{2} \int_{\mathbb{R}^{n}} \int_{\mathbb{R}^{n}} \frac{|u(x)-u(y)|^{2}}{|x-y|^{n+2 s}} d y d x+\int_{\Omega} c u^{2} d x
$$


it is easy to see that $I$ is bounded from below and coercive. In addition, let $w, v \in X_{0}$ with $w \neq v$. A straightforward calculation yields that

$$
\begin{aligned}
\left\langle I^{\prime}(w)-I^{\prime}(v), w-v\right\rangle & =\frac{c_{n, s}}{2} \int_{\mathbb{R}^{2 n}} \frac{|(w-v)(x)-(w-v)(y)|^{2}}{|x-y|^{n+2 s}} d y d x+\int_{\Omega} c(x)(w-v)^{2} d x \\
& \geq \lambda_{s}\|w-v\|_{L^{2}(\Omega)}^{2}>0 .
\end{aligned}
$$

Namely, $I$ is strictly convex, and it follows from [13] that $I$ has a unique minimizer still denoted by $u$. That is, the boundary value problem has a unique solution. Given that $f \in L^{2}(\Omega)$ with $f \geq 0$. Let $u_{-}=\min \{u, 0\}$, Clearly, $u_{-} \in H_{0}^{s}(\Omega)$. We show that $u_{-} \equiv 0$,

$$
\begin{aligned}
& \left\langle I^{\prime}(u), u_{-}\right\rangle \\
& =\frac{c_{n, s}}{2} \int_{\mathbb{R}^{2 n}} \frac{(u(x)-u(y))\left(u_{-}(x)-u_{-}(y)\right)}{|x-y|^{n+2 s}} d y d x-\int_{\Omega} c(x)\left(u_{-}\right)^{2} d x-\int_{\Omega} f u_{-} d x \\
& =-\frac{c_{n, s}}{2} \int_{\mathbb{R}^{2 n}} \frac{\left|u_{-}(x)-u_{-}(y)\right|^{2}}{|x-y|^{n+2 s}} d y d x \\
& \quad+\frac{c_{n, s}}{2} \int_{\mathbb{R}^{2 n}} \frac{\left(u_{+}(x)-u_{+}(y)\right)\left(u_{-}(x)-u_{-}(y)\right)}{|x-y|^{n+2 s}} d y d x-\int_{\Omega} c(x)\left(u_{-}\right)^{2} d x-\int_{\Omega} f u_{-} d x \\
& =-\frac{c_{n, s}}{2} \int_{\mathbb{R}^{2 n}} \frac{\left|u_{-}(x)-u_{-}(y)\right|^{2}}{|x-y|^{n+2 s}} d y d x-c_{n, s} \int_{\mathbb{R}^{2 n}} \frac{u_{+}(x) u_{-}(y)}{|x-y|^{n+2 s}} d y d x-\int_{\Omega} c(x)\left(u_{-}\right)^{2} d x \\
& \quad-\int_{\Omega} f u_{-} d x=0 .
\end{aligned}
$$

Here $\mathbb{R}^{2 n}:=\mathbb{R}^{n} \times \mathbb{R}^{n}$. Hence,

$$
\lambda_{s}\left\|u_{-}\right\|_{L^{2}(\Omega)}^{2} \leq-c_{n, s} \int_{\mathbb{R}^{2 n}} \frac{u_{+}(x) u_{-}(y)}{|x-y|^{n+2 s}} d y d x-\int_{\Omega} f u_{-} d x \leq 0 .
$$

This implies that $u_{-}=0$. Finally, we show (iv) implies (i). Choose any $f \in C(\bar{\Omega})$ with $f \gtrless 0$. Then the boundary value problem has a unique solution $u_{f} \in H_{0}^{s}(\Omega)$ and $u_{f} \geqslant 0$. Again, Proposition 2.1 and Proposition 1.1 of [21] imply that $u_{f} \in C^{s}\left(\mathbb{R}^{n}\right)$. In particular, we have $u_{f}>0$ in $\Omega$. Thus $u_{f}$ is a strict super-solution of $\left\{L_{c}, \mathcal{D}, \Omega\right\}$. Namely, (ii) holds, and hence (i) follows.

Remark 2. Assume that $\lambda_{s}>0$. Let $u_{f}$ be the unique weak solution of (2.1). Then straightforward calculation shows that $\left\|u_{f}\right\|_{L^{2}(\Omega)} \leq \lambda_{s}^{-1}\|f\|_{L^{2}(\Omega)}$.

Proposition 2.4. Assume that $\Omega \subset \mathbb{R}^{n}$ is a bounded domain with Lipschitz boundary. Let $\lambda_{s}$ be the principal eigenvalue of $\left\{L_{c}, \mathcal{D}, \Omega\right\}$. Then

$$
\lambda_{s} \geq \frac{1}{r^{2 s}} \frac{4^{s} \Gamma(s+1) \Gamma\left(s+\frac{n}{2}\right)(2 s+2)(2 s+n)(6-2 s)}{\Gamma\left(\frac{n}{2}\right)[12 n+(16-2 n) s]}+\inf _{x \in \Omega} c,
$$

where $r>0$ is the positive number such that $|\Omega|=\left|B_{r}(0)\right|$. 
Proof. The idea of the proof is similar to that of the proof for Proposition 8.6 of [17]. We shall present a sketch. Let $\psi$ be an eigenfunction corresponding to $\lambda_{s}$ with $\|\psi\|_{L^{2}(\Omega)}=1$, then the Faber-Krahn inequality of [5] along with Proposition 2.2 imply that

$$
\lambda_{s} \geq \frac{c_{n, s}}{2} \int_{\mathbb{R}^{n}} \int_{\mathbb{R}^{n}} \frac{|\psi(x)-\psi(y)|^{2}}{|x-y|^{n+2 s}} d y d x+\inf _{x \in \Omega} c \geq\left(\frac{\left|B_{r}(0)\right|}{|\Omega|}\right)^{\frac{2 s}{n}} \lambda_{1}\left(B_{r}\right)+\inf _{x \in \Omega} c,
$$

where $\lambda_{1}\left(B_{r}\right)$ is the principal eigenvalue of $\left\{(-\Delta)^{s}, \mathcal{D}, B_{r}(0)\right\}$. Note that $\lambda_{1}\left(B_{r}\right)=r^{-2 s} \lambda_{1}\left(B_{1}\right)$. Now choose $r>0$ such that $\left|B_{r}(0)\right|=|\Omega|$, the desired conclusion follows from Lemma 9 of [12]. The proof is completed.

Proposition 2.5. Let $\Omega_{i}(i=1,2) \subset \mathbb{R}^{n}$ be two bounded open domains with $\partial \Omega_{i} \in \mathcal{C}^{1}$ such that $\Omega_{2} \subseteq \Omega_{1}$. Let $\lambda_{\Omega_{i}}$ be the principal eigenvalues of $\left\{L_{c}, \mathcal{D}, \Omega_{i}\right\}$, respectively. Then $\lambda_{\Omega_{1}} \leq \lambda_{\Omega_{2}}$. In case that $\Omega_{2} \subsetneq \Omega_{1}$, then $\lambda_{\Omega_{1}}<\lambda_{\Omega_{2}}$. Let $\lambda_{c_{i}}$ be the principal eigenvalue of $\left\{L_{c_{i}}, \mathcal{D}, \Omega\right\}$ with $c_{i} \in L^{\infty}(\Omega) ;(i=1,2)$. Assume that $c_{1} \leq c_{2}$. Then $\lambda_{c_{1}} \leq \lambda_{c_{2}}$. In case that $c_{i} \lesseqgtr c_{2}$, then $\lambda_{c_{1}}<\lambda_{c_{2}}$. Furthermore, $\lambda_{c}$ is Lipschitz continuous with respect to $c$.

Proof. Clearly, $H_{0}^{s}\left(\Omega_{2}\right) \subseteq H_{0}^{s}\left(\Omega_{1}\right)$. Thus, it follows from Proposition 2.2 that $\lambda_{\Omega_{2}} \geq \lambda_{\Omega_{1}}$. In case that $\Omega_{2} \subsetneq \Omega_{1}$, let $\varphi_{1}$ be a positive eigenfunction associated with $\lambda_{\Omega_{1}}$, then we have $L_{c} \varphi_{1}-$ $\lambda_{\Omega_{1}} \varphi_{1}=0$ in $\Omega_{2}$ and $\varphi_{1} \gtrless 0$ in $\mathbb{R}^{n} \backslash \Omega_{2}$. Namely, $\varphi_{1}$ is a strict super-solution of $\left\{L_{c}-\right.$ $\left.\lambda_{\Omega_{1}}, \mathcal{D}, \Omega_{2}\right\}$. As $\lambda_{\Omega_{2}}-\lambda_{\Omega_{1}}$ is the principal eigenvalue of $\left\{L_{c}-\lambda_{\Omega_{1}}, \mathcal{D}, \Omega_{2}\right\}$, Theorem 2.1 yields that $\lambda_{\Omega_{2}}>\lambda_{\Omega_{1}}$. The monotonicity of $\lambda_{c}$ with respect to $c$ is also an immediate consequence of Theorem 2.1. We now proceed to show the Lipschitz continuity of $\lambda_{c}$ with respect to $c$. Notice that

$$
\int_{\mathbb{R}^{2 n}} \frac{\left[\varphi_{i}(x)-\varphi_{i}(y)\right]\left[\varphi_{j}(x)-\varphi_{j}(y)\right]}{|x-y|^{n+2 s}} d y d x+\int_{\Omega} c_{i} \varphi_{i} \varphi_{j} d x=\int_{\Omega} \lambda_{c_{i}} \varphi_{i} \varphi_{j} d x .
$$

Here either $i=1$ and $j=2$, or $i=2$ and $j=1$, again $\lambda_{c_{i}}$ is the principal eigenvalue of $L_{c_{i}}$ in $\Omega$. By subtracting the two equations, we have

$$
\left(\lambda_{c_{1}}-\lambda_{c_{2}}\right)=\frac{\int_{\Omega}\left(c_{1}-c_{2}\right) \varphi_{1} \varphi_{2} d x}{\int_{\Omega} \varphi_{1} \varphi_{2} d x}
$$

Therefore,

$$
\left|\lambda_{c_{1}}-\lambda_{c_{2}}\right| \leq \frac{\int_{\Omega}\left|c_{1}-c_{2}\right| \varphi_{1} \varphi_{2} d x}{\int_{\Omega} \varphi_{1} \varphi_{2} d x} \leq\left\|c_{1}-c_{2}\right\|_{L^{\infty}(\Omega)} .
$$

Namely, $\lambda_{c}$ is Lipschitz continuous with respect to $c$. The proof is completed.

Proposition 2.6. Assume that $\Omega \subset \mathbb{R}^{n}$ is a connected and bounded domain with $\partial \Omega \in \mathcal{C}^{1}$. Let $\Omega_{i} \subset \subset \Omega$ be open subsets of $\Omega$ with smooth boundary satisfying $\Omega_{i} \subset \Omega_{i+1}$ and $\bigcup_{j=1}^{\infty} \Omega_{j}=\Omega$. Let $\phi \in C_{0}^{\infty}(\Omega)$. Then there exists a sequence $\left\{\phi_{j}\right\}_{j=1}^{\infty}$ with $\phi_{j} \in C_{0}^{\infty}\left(\Omega_{j}\right)$ such that $\lim _{j \rightarrow \infty} \| \phi_{j}-$ $\phi \|_{H_{0}^{s}(\Omega)}=0$. 
Proof. Denote that $A_{\delta}:=\left\{x \in \Omega \mid \operatorname{dist}(x, \partial \Omega)<\frac{1}{\delta}\right\}$, where $\delta>0$. Assume without loss of generality that $\Omega_{j} \subseteq \Omega \backslash A_{4 j}$ and that $\Omega \backslash A_{2 j} \subseteq \Omega_{j}$. Following [17], we choose $w_{j} \in C_{0}^{\infty}(\Omega)$ such that $0 \leq w_{j} \leq 1, w_{j}=1$ in $\bar{\Omega} \backslash A_{j}$, and $w_{j}=0$ in $A_{2 j}$. Moreover, $\left\|\nabla w_{j}\right\|_{C(\bar{\Omega})} \leq C j$ for some positive constant $C$ independent of $j$. Now write $\phi_{j}=w_{j} \phi$. Clearly, $\phi_{j} \in C_{0}^{\infty}\left(\Omega_{j}\right)$. It is easy to see that $\phi_{j} \rightarrow \phi$ in $L^{2}(\Omega)$ as $j \rightarrow \infty$. Notice that $\left(\phi_{j}-\phi\right)(x)-\left(\phi_{j}-\phi\right)(y)=0$ whenever $(x, y) \in\left(\Omega \backslash A_{j}\right) \times\left(\Omega \backslash A_{j}\right),\left(\Omega \backslash A_{j}\right) \times \Omega^{c}$, and $\Omega^{c} \times \Omega^{c}$. Hence, we have

$$
\begin{aligned}
\int_{\mathbb{R}^{2 n}} & \frac{\left|\left(\phi_{j}-\phi\right)(x)-\left(\phi_{j}-\phi\right)(y)\right|^{2}}{|x-y|^{n+2 s}} d y d x \\
= & 2 \int_{A_{j} \times\left(\Omega \backslash A_{j}\right)}+\int_{A_{j} \times A_{j}} \frac{\left|\left(\phi_{j}-\phi\right)(x)-\left(\phi_{j}-\phi\right)(y)\right|^{2}}{|x-y|^{n+2 s}} d y d x \\
& +2 \int_{A_{j} \times \Omega^{c}} \frac{\left|\left(\phi_{j}-\phi\right)(x)-\left(\phi_{j}-\phi\right)(y)\right|^{2}}{|x-y|^{n+2 s}} d y d x .
\end{aligned}
$$

Let $\varrho=\sup \{|x-y| \mid x, y \in \bar{\Omega}\}$. By Fubini's theorem and mean value theorem, we find that

$$
\begin{aligned}
\int_{A_{j} \times \Omega} & \frac{\left|\left(\phi_{j}-\phi\right)(x)-\left(\phi_{j}-\phi\right)(y)\right|^{2}}{|x-y|^{n+2 s}} d y d x \\
\leq & \int_{A_{j} \times \Omega} 2 \frac{\left|\phi_{j}(x)-\phi_{j}(y)\right|^{2}+|\phi(x)-\phi(y)|^{2}}{|x-y|^{n+2 s}} d y d x \\
\leq & 4 \int_{A_{j}} d x \int_{\Omega} \frac{|\phi(x)|^{2}\left|w_{j}(x)-w_{j}(y)\right|^{2}+\left|w_{j}(y)\right|^{2}|\phi(x)-\phi(y)|^{2}}{|x-y|^{n+2 s}} d y \\
& +2 \int_{A_{j} \times \Omega} \frac{|\phi(x)-\phi(y)|^{2}}{|x-y|^{n+2 s}} d y d x \\
\leq & 4 \int_{A_{j}} d x \int_{B_{\varrho}(x)} \frac{|\phi(x)|^{2}\left|w_{j}(x)-w_{j}(y)\right|^{2}}{|x-y|^{n+2 s}} d y+6 \int_{A_{j}} d x \int_{B_{\varrho}(x)} \frac{|\phi(x)-\phi(y)|^{2}}{|x-y|^{n+2 s}} d y \\
\leq & 4 \omega_{n} \int_{A_{j}} j^{2}|\phi(x)|^{2} d x \int_{0}^{\varrho} \frac{r^{n+1}}{r^{(n+2 s)}} d r+6 \omega_{n} \int_{A_{j}}\|\nabla \phi\|_{L^{\infty}(\Omega)}^{2} d x \int_{0}^{\varrho} \frac{r^{n+1}}{r^{(n+2 s)}} d r \\
\leq & 4 \omega_{n} \varrho^{2(1-s)}\|\nabla \phi\|_{L^{2}\left(A_{j}\right)}^{2}+6 \omega_{n} \varrho^{2(1-s)}\|\nabla \phi\|_{L^{\infty}(\Omega)}^{2}\left|A_{j}\right| .
\end{aligned}
$$

Here $\omega_{n}=\frac{2 \pi^{\frac{n}{2}}}{\Gamma(n / 2)}$, and we used the fact that $\|\phi\|_{L^{2}\left(A_{j}\right)} \leq \frac{1}{j}\|\nabla \phi\|_{L^{2}\left(A_{j}\right)}($ see Remark 4.1 of [17]). Next let $\Omega_{1}^{c}=\left\{x \in \Omega^{c} \mid \operatorname{dist}(x, \partial \Omega) \leq 1\right\}$, and $\iota=\sup \left\{|x-y| \mid x, y \in \overline{A_{j} \cup \Omega_{1}^{c}}\right\}$. Straightforward calculation shows that

$$
\begin{aligned}
\int_{A_{j} \times \Omega^{c}} & \frac{\left|\left(\phi_{j}-\phi\right)(x)-\left(\phi_{j}-\phi\right)(y)\right|^{2}}{|x-y|^{n+2 s}} d y d x \\
& =\int_{A_{j} \times \Omega_{1}^{c}}+\int_{A_{j} \times\left(\Omega^{c} \backslash \Omega_{1}^{c}\right)} \frac{\left|\left(\phi_{j}-\phi\right)(x)-\left(\phi_{j}-\phi\right)(y)\right|^{2}}{|x-y|^{n+2 s}} d y d x \\
& \leq \omega_{n} \iota^{2(1-s)}\left[4\|\nabla \phi\|_{L^{2}\left(A_{j}\right)}^{2}+6\|\nabla \phi\|_{L^{\infty}(\Omega)}^{2}\left|A_{j}\right|\right]
\end{aligned}
$$




$$
\begin{gathered}
+\int_{A_{j} \times\left(\Omega^{c} \backslash \Omega_{1}^{c}\right)} \frac{\left|\left(\phi_{j}-\phi\right)(x)-\left(\phi_{j}-\phi\right)(y)\right|^{2}}{|x-y|^{n+2 s}} d y d x \\
\leq \omega_{n} \iota^{2(1-s)}\left[4\|\nabla \phi\|_{L^{2}\left(A_{j}\right)}^{2}+6\|\nabla \phi\|_{L^{\infty}(\Omega)}^{2}\left|A_{j}\right|\right]+4 \omega_{n} \int_{A_{j}}\|\phi\|_{L^{\infty}(\Omega)}^{2} d x \int_{1}^{\infty} \frac{r^{n-1}}{r^{n+2 s}} d r \\
\leq \omega_{n} \iota^{2(1-s)}\left[4\|\nabla \phi\|_{L^{2}\left(A_{j}\right)}^{2}+6\|\nabla \phi\|_{L^{\infty}(\Omega)}^{2}\left|A_{j}\right|\right]+4 \omega_{n}\|\phi\|_{L^{\infty}(\Omega)}^{2}\left|A_{j}\right| .
\end{gathered}
$$

Since $\|\nabla \phi\|_{L^{2}\left(A_{j}\right)} \rightarrow 0$, and $\left|A_{j}\right| \rightarrow 0$ as $j \rightarrow \infty$, we readily conclude that $\lim _{j \rightarrow \infty} \| \phi_{j}-$ $\phi \|_{H_{0}^{s}(\Omega)}=0$. The proof is completed.

Theorem 2.2. Assume that $\Omega \subset \mathbb{R}^{n}$ is a connected and bounded domain with $\partial \Omega \in \mathcal{C}^{1}$. Let $\lambda_{\Omega_{i}}$ be the principal eigenvalue of $\left\{L_{c}, \mathcal{D}, \Omega_{i}\right\}$, where $\Omega_{i} \subset \mathbb{R}^{n}$ are a sequence of bounded domains with smooth boundary. (i) Assume that $\Omega_{i} \subset \subset \Omega$ satisfying $\Omega_{i} \subset \Omega_{i+1}$ and $\bigcup_{j=1}^{\infty} \Omega_{j}=\Omega$. Then $\lim _{j \rightarrow \infty} \lambda_{\Omega_{j}}=\lambda_{\Omega}$. (ii) Assume that $\Omega_{i} \subset \subset \Omega$ satisfying $\Omega_{i+1} \subset \Omega_{i}$ and $\bigcap_{j=1}^{\infty} \Omega_{j}=\Omega_{0} \subset \Omega$. Then $\lim _{j \rightarrow \infty} \lambda_{\Omega_{j}}=\lambda_{\Omega_{0}}$.

Proof. To establish (i), we let $\varphi_{i}$ be a positive eigenfunction corresponding to $\lambda_{\Omega_{i}}$. Namely,

$$
(-\Delta)^{s} \varphi_{i}+c(x) \varphi_{i}=\lambda_{\Omega_{i}} \varphi_{i}
$$

in $\Omega_{i}$ and $\varphi_{i}=0$ in $\Omega^{c}$. As a result of Proposition 2.5, there holds that $\lambda_{i} \geq \lambda_{i+1}>\lambda_{\Omega}$. Hence $\lambda_{\Omega_{i}}$ are bounded and $\lim _{i \rightarrow \infty} \lambda_{\Omega_{i}}$ exists. Assume without loss of generality that $\left\|\varphi_{i}\right\|_{L^{2}(\Omega)}=1$. Then, in view of Proposition 2.1, we have $\left\|\varphi_{i}\right\|_{C^{s}\left(\mathbb{R}^{n}\right)} \leq C$ for some positive constant $C$. Since $\varphi_{i}=0$ in $\Omega_{i}^{c}$, it follows from Ascoli-Arzelà theorem that there exists $\varphi^{*} \in C\left(\mathbb{R}^{n}\right)$ for which $\lim _{i \rightarrow \infty}\left\|\varphi_{i}-\varphi^{*}\right\|_{C\left(\mathbb{R}^{n}\right)}=0$. Clearly, $\varphi^{*}=0$ in $\Omega^{c}$ and $\varphi^{*} \gtrless 0$ in $\Omega$. We next show that $\lim _{i \rightarrow \infty}\left\|\varphi_{i}-\varphi^{*}\right\|_{H_{0}^{s}(\Omega)}=0$. To this end, we show that $\left\{\varphi_{i}\right\}$ is a Cauchy sequence of $H_{0}^{s}(\Omega)$. Note that

$$
\begin{aligned}
& \int_{\mathbb{R}^{2 n}} \frac{\left|\left(\varphi_{j}-\varphi_{i}\right)(x)-\left(\varphi_{j}-\varphi_{i}\right)(y)\right|^{2}}{|x-y|^{n+2 s}} d y d x=\int_{\mathbb{R}^{2 n}} \frac{\left|\varphi_{j}(x)-\varphi_{j}(y)\right|^{2}}{|x-y|^{n+2 s}} d y d x \\
& +\int_{\mathbb{R}^{2 n}} \frac{\left|\varphi_{i}(x)-\varphi_{i}(y)\right|^{2}}{|x-y|^{n+2 s}} d y d x-2 \int_{\mathbb{R}^{2 n}} \frac{\left[\varphi_{j}(x)-\varphi_{j}(y)\right]\left[\varphi_{i}(x)-\varphi_{i}(y)\right]}{|x-y|^{n+2 s}} d y d x .
\end{aligned}
$$

Assume that $j>i$, then $\varphi_{i} \in H_{0}^{s}\left(\Omega_{j}\right)$, and

$$
\begin{aligned}
\frac{c_{n, s}}{2} & \int_{\mathbb{R}^{2 n}} \frac{\left|\left(\varphi_{j}-\varphi_{i}\right)(x)-\left(\varphi_{j}-\varphi_{i}\right)(y)\right|^{2}}{|x-y|^{n+2 s}} d y d x \\
& =\int_{\Omega_{j}}\left(\lambda_{\Omega_{j}}-c\right) \varphi_{j}^{2} d x+\int_{\Omega_{i}}\left(\lambda_{\Omega_{i}}-c\right) \varphi_{i}^{2} d x-2 \int_{\Omega_{j}}\left(\lambda_{\Omega_{j}}-c\right) \varphi_{j} \varphi_{i} d x \\
& \leq\left\|\varphi_{j}-\varphi_{i}\right\|_{L^{2}(\Omega)}\left\|\left(\lambda_{\Omega_{j}}-c\right) \varphi_{j}\right\|_{L^{2}(\Omega)}+\left\|\left(\lambda_{\Omega_{j}}-c\right) \varphi_{j}-\left(\lambda_{\Omega_{i}}-c\right) \varphi_{i}\right\|_{L^{2}(\Omega)}\left\|\varphi_{i}\right\|_{L^{2}(\Omega)} .
\end{aligned}
$$

Thus, $\left\{\varphi_{i}\right\}$ is a Cauchy sequence in $H_{0}^{s}(\Omega)$ as $\varphi_{i}$ converges to $\varphi^{*}$ in $L^{2}(\Omega)$ and $\lim _{i \rightarrow \infty} \lambda_{\Omega_{i}}$ exists. In particular, in view of Poincaré- Sobolev inequality, we have $\lim _{i \rightarrow \infty}\left\|\varphi_{i}-\varphi^{*}\right\|_{H_{0}^{s}(\Omega)}=0$. 
Given $\phi \in C_{0}^{\infty}(\Omega)$, in view of Proposition 2.6, there exists $\left\{\phi_{i}\right\}$ with $\phi_{i} \in C_{0}^{\infty}\left(\Omega_{i}\right)$ such that $\phi_{i}$ converges to $\phi$ in $H_{0}^{s}(\Omega)$ as $i \rightarrow \infty$. Then

$$
\frac{c_{n, s}}{2} \int_{\mathbb{R}^{2 n}} \frac{\left[\varphi_{i}(x)-\varphi_{i}(y)\right]\left[\phi_{i}(x)-\phi_{i}(y)\right]}{|x-y|^{n+2 s}} d y d x+\int_{\Omega} c(x) \varphi_{i} \phi_{i} d x=\lambda_{\Omega_{i}} \int_{\Omega} \varphi_{i} \phi_{i} d x .
$$

By passing the limits in the above equation, we find that

$$
\frac{c_{n, s}}{2} \int_{\mathbb{R}^{2 n}} \frac{\left[\varphi^{*}(x)-\varphi^{*}(y)\right][\phi(x)-\phi(y)]}{|x-y|^{n+2 s}} d y d x+\int_{\Omega} c(x) \varphi^{*} \phi_{i} d x=\lambda^{*} \int_{\Omega} \varphi^{*} \phi_{i} d x .
$$

Here $\lambda^{*}=\lim \lambda_{\Omega_{i}}$. This yields that $\lambda^{*}$ is an eigenvalue of $\left\{L_{c}, \mathcal{D}, \Omega\right\}$ and $L_{c} \varphi^{*}=\lambda^{*} \varphi^{*}$ in $\Omega$. Again, Proposition 2.3 implies that $\varphi^{*}>0$ in $\Omega$. Thus $\lambda^{*}=\lambda_{\Omega}$. We now proceed to show (ii). With slight abuse of the notations, we still let $\varphi_{i}$ be the positive eigenfunction corresponding to the eigenvalue $\lambda_{i}$ with $\left\|\varphi_{i}\right\|_{L^{2}(\Omega)}=1$. It is easy to see that $\lambda_{i} \leq \lambda_{i+1}<\lambda_{\Omega}$. In particular, it follows from the same reasoning that $\lim _{i \rightarrow \infty}\left\|\varphi_{i}-\varphi_{*}\right\|_{H^{s}\left(\mathbb{R}^{n}\right)}=0$ and $\lim _{i \rightarrow \infty}\left\|\varphi_{i}-\varphi_{*}\right\|_{C\left(\mathbb{R}^{n}\right)}=0$ for some $\varphi_{*} \in H_{0}^{s}\left(\Omega_{0}\right) \cap C\left(\mathbb{R}^{n}\right)$. Note that $H_{0}^{s}\left(\Omega_{0}\right) \subset H_{0}^{s}\left(\Omega_{i}\right)$. Let $\phi \in H_{0}^{s}\left(\Omega_{0}\right)$, then

$$
\frac{c_{n, s}}{2} \int_{\mathbb{R}^{2 n}} \frac{\left[\varphi_{i}(x)-\varphi_{i}(y)\right][\phi(x)-\phi(y)]}{|x-y|^{n+2 s}} d y d x+\int_{\Omega_{0}} c(x) \varphi_{i} \phi_{i} d x=\lambda_{\Omega_{i}} \int_{\Omega_{0}} \varphi_{i} \phi d x .
$$

Let $\lambda_{*}=\lim _{i \rightarrow \infty} \lambda_{\Omega_{i}}$. By passing the limits in the above equation, we find that

$$
\frac{c_{n, s}}{2} \int_{\mathbb{R}^{2 n}} \frac{\left[\varphi_{*}(x)-\varphi_{*}(y)\right][\phi(x)-\phi(y)]}{|x-y|^{n+2 s}} d y d x+\int_{\Omega_{0}} c(x) \varphi_{*} \phi d x=\lambda_{*} \int_{\Omega_{0}} \varphi_{*} \phi d x .
$$

Again, the same argument as that for (i) yields that $\lambda_{*}=\lambda_{\Omega_{0}}$. The proof is completed.

Motivated by [2] and [17], a point-wise min-max characterization of $\lambda_{s}$ can be given as follows

Proposition 2.7. Suppose that $\Omega \subset \mathbb{R}^{n}$ is a connected and bounded domain with $\partial \Omega \in \mathcal{C}^{1}$. Set $P:=\left\{u \in C(\bar{\Omega}) \cap L_{\text {loc }}^{\infty}\left(\mathbb{R}^{n}\right) \mid \phi \geqslant 0\right.$ in $\left.\mathbb{R}^{n}, L_{c} \phi \in L^{\infty}(\Omega)\right\}$. Let $\lambda_{s}^{\prime}:=\sup \{\lambda \mid \exists \phi \in$ $P, L_{c} \phi \geq \lambda \phi$ in $\left.\Omega\right\}$. Then $\lambda_{s}^{\prime}=\lambda_{s}$, where $\lambda_{s}$ is the principle eigenvalue of $\left\{L_{c}, \mathcal{D}, \Omega\right\}$. In addition, $\lambda_{s}=\sup _{\phi \in P} \inf _{\Omega}\left(\frac{L_{c} \phi}{\phi}\right)$. Furthermore, let $\widetilde{P}:=\{\phi \in P \mid \phi>0$ in $\bar{\Omega}\}$, and $\lambda_{s}^{\prime \prime}:=\sup \{\lambda \mid$ $\exists \phi \in \widetilde{P}, L_{c} \phi \geq \lambda \phi$ in $\left.\Omega\right\}$. Then $\lambda_{s}^{\prime \prime}=\lambda_{s}$. Additionally, $\lambda_{s}=\sup _{\phi \in \widetilde{P}} \inf _{\Omega}\left(\frac{L_{c} \phi}{\phi}\right)$.

Proof. It is obvious that $\lambda_{s}^{\prime} \geq \lambda_{s}$. Assume to the contrary that $\lambda_{s}^{\prime}>\lambda_{s}$. Let $\eta=\frac{\lambda_{s}^{\prime}-\lambda_{s}}{2}$. Then, there exists $\psi \in C\left(\mathbb{R}^{n}, \mathbb{R}^{+}\right)$with $\psi>0$ in $\Omega$ such that $L_{c} \psi-\lambda_{s} \psi \geq \eta \psi>0$ in $\Omega$. Hence, it follows from Theorem 2.1 that the principal eigenvalue of $\left\{L_{c}-\lambda_{s} I, \mathcal{D}, \Omega\right\}$ is strictly positive, which is a contradiction. Thus, $\lambda_{s}^{\prime}=\lambda_{s}$. To show that $\lambda_{s}=\sup _{\phi \in P} \inf _{\Omega}\left(\frac{L_{c} \phi}{\phi}\right)$, we fix $\lambda<\lambda_{s}$. Then Theorem 2.1 implies that the boundary value problem: $\left(L_{c}-\lambda\right) u=1$ in $\Omega$ and $u=0$ in $\Omega^{c}$ has a unique positive solution $\psi_{\lambda} \in C^{s}\left(\mathbb{R}^{n}\right)$. Clearly, $\inf _{\Omega}\left(L_{c} \psi_{\lambda} / \psi_{\lambda}\right)>\lambda$. Hence $\lambda<$ 
$\sup _{\phi \in P} \inf _{\Omega}\left(\frac{L_{c} \phi}{\phi}\right)$. Since this is true for any $\lambda<\lambda_{s}$, it follows that $\lambda_{s} \leq \sup _{\phi \in P} \inf _{\Omega}\left(\frac{L_{c} \phi}{\phi}\right)$. On the other hand, by means of the same argument given above, we can show that $\lambda_{s}^{\prime}=\lambda_{s}$. We now prove the last part. Given any $\lambda<\lambda_{s}$, Theorem 2.1 and Proposition 2.6 imply that there exists $\Omega_{\lambda} \subset \mathbb{R}^{n}$ with $\Omega \subset \subset \Omega_{\lambda}$ such that $\left(L_{c}-\lambda\right) u=1$ in $\Omega_{\lambda}$ and $u=0$ in $\Omega_{\lambda}^{c}$ has a unique positive solution $\psi_{\lambda} \in C^{s}\left(\mathbb{R}^{n}\right)$. Clearly, $\psi_{\lambda} \in \widetilde{P}$. Thus, it follows from the same reasoning that $\lambda_{s}^{\prime \prime}=\lambda_{s}=\sup _{\phi \in \widetilde{P}} \inf _{\Omega}\left(\frac{L_{c} \phi}{\phi}\right)$. The proof is completed.

We next consider the existence and multiplicity of principal eigenvalues for the linear weighted boundary value problem

$$
\left\{\begin{array}{l}
L_{c} u=\lambda a(x) u, \quad x \in \Omega, \\
u=0, \quad x \in \mathbb{R}^{n} \backslash \Omega,
\end{array}\right.
$$

where $a \in L^{\infty}(\Omega)$. Let $\mu(\lambda)$ be the principal eigenvalue of $\left\{L_{c}-\lambda a, \mathcal{D}, \Omega\right\}$. Following [17], $\lambda_{s}$ is referred to as a principal eigenvalue of (2.4) if $\mu\left(\lambda_{s}\right)=0$. In view of Proposition 2.1, it is easy to see that $\mu\left(t \lambda_{1}+(1-t) \lambda_{2}\right) \geq t \mu\left(\lambda_{1}\right)+(1-t) \mu\left(\lambda_{2}\right)$ for any $t \in[0,1]$ and $\lambda_{1}, \lambda_{2} \in \mathbb{R}$, that is, $\mu(\lambda)$ is concave with respect to $\lambda$. In addition, by invoking the same argument as that given in [17] (see Theorem 9.1), we infer that $\mu(\lambda)$ is analytic in $\lambda \in \mathbb{R}$. The following results, Theorem 2.3 and Theorem 2.4, are given without proofs because the proofs are the same as those for theorem 9.3 and Theorem 9.4 in [17] in the presence of Proposition 2.2 and Proposition 2.5.

Theorem 2.3. Assume that $\Omega \subset \mathbb{R}^{n}$ is a bounded domain with Lipschitz boundary. Let $\mu(\lambda)$ be the principal eigenvalue of $\left\{L_{c}-\lambda a, \mathcal{D}, \Omega\right\}$. (i) Suppose that there exists $\Omega_{+} \subset \Omega$ such that $a \geqslant 0$ in $\Omega_{+}$. Then $\lim _{\lambda \rightarrow \infty} \mu(\lambda)=-\infty$. In addition, Assume that $a \gtrless 0$ in $\Omega$. Then (2.4) has a principal eigenvalue if and only if $\lim _{\lambda \rightarrow-\infty} \mu(\lambda)>0$. Moreover, the principal eigenvalue is unique if it exists. (ii) Suppose that there exists $\Omega_{-} \subset \Omega$ such that $a \lesseqgtr 0$ in $\Omega_{-}$. Then $\lim _{\lambda \rightarrow \infty} \mu(\lambda)=\infty$. In addition, Assume that $a \lesseqgtr 0$ in $\Omega$. Then (2.4) has a principal eigenvalue if and only if $\lim _{\lambda \rightarrow-\infty} \mu(\lambda)<0$. Moreover, the principal eigenvalue is unique if it exists.

Theorem 2.4. Assume that $\Omega \subset \mathbb{R}^{n}$ is a bounded domain with Lipschitz boundary. Suppose a $\in$ $L^{\infty}(\Omega)$ changes sign in the sense that there exist $\Omega_{ \pm} \subset \Omega$ such that $a \gtrless 0$ in $\Omega_{+}$, and $a \lesseqgtr 0$ in $\Omega_{-}$. Let $\mu(\lambda)$ be the principal eigenvalue of $\left\{L_{c}-\lambda a, \mathcal{D}, \Omega\right\}$. Let $\mu\left(\lambda^{*}\right)=\max _{\lambda \in \mathbb{R}} \mu(\lambda)$ for some $\lambda^{*} \in \mathbb{R}$. Then (i) (2.4) has no principal eigenvalues if $\mu\left(\lambda^{*}\right)<0$. (ii) In case that $\mu\left(\lambda^{*}\right)=0,(2.4)$ has one and only one principal eigenvalue, which is $\lambda^{*}$. (iii) (2.4) possesses exactly two principal eigenvalues $\lambda_{s}^{-}(a)$ and $\lambda_{s}^{+}(a)$ with $\lambda_{s}^{-}(a)<\lambda_{s}^{+}(a)$ provided that $\mu\left(\lambda^{*}\right)>0$. In particular, under the assumption that $\mu\left(\lambda^{*}\right)>0$, there hold (a) $\lambda_{s}^{-}(a)<0<\lambda_{s}^{+}(a)$ if $\mu(0)>0$. (b) $0=\lambda_{s}^{-}(a)<\lambda_{s}^{+}(a)$ if $\mu(0)=0$ and $\mu^{\prime}(0)>0$. (c) $\lambda_{s}^{-}(a)<\lambda_{s}^{+}(a)=0$ if $\mu(0)=0$ and $\mu^{\prime}(0)<0$. (d) $0<\lambda_{s}^{-}(a)<\lambda_{s}^{+}(a)$ if $\mu(0)<0$ and $\mu^{\prime}(0)>0$. (e) $\lambda_{s}^{-}(a)<\lambda_{s}^{+}(a)<0$ if $\mu(0)<0$ and $\mu^{\prime}(0)<0$. 
Corollary 2.5. Let all of the assumptions of Theorem 2.4 are satisfied. Suppose that $\mu(0)>0$. Let $\lambda_{s}^{-}(a)<0<\lambda_{s}^{+}(a)$ be the principal eigenvalues of (2.4) given in Theorem 2.4. Then, there holds that

$$
\lambda_{s}^{+}(a)=\inf _{\left\{u \in H_{0}^{s}(\Omega) \mid I_{+}(u)=1\right\}} J(u), \quad \lambda_{s}^{-}(a)=-\inf _{\left\{u \in H_{0}^{s}(\Omega) \mid I_{-}(u)=1\right\}} J(u),
$$

where

$$
J(u)=\frac{c_{n, s}}{2} \int_{\mathbb{R}^{n}} \int_{\mathbb{R}^{n}} \frac{|u(x)-u(y)|^{2}}{|x-y|^{n+2 s}} d y d x+\int_{\Omega} c(x) u^{2} d x, \quad I_{ \pm}(u)=\int_{\Omega} \pm a(x) u^{2} d x .
$$

Proof. Let $\theta \in \mathbb{R}$. It is easy to see that $J$ is bounded from below, weakly lower semi-continuous, and coercive on the manifolds $I_{ \pm}(u)=\theta$. Write $\lambda_{ \pm}=\inf _{\left\{u \in H_{0}^{s}(\Omega) \mid I_{ \pm}(u)=1\right\}} J(u)$. In view of Theorem 1.1.1 of [9], $\lambda_{ \pm}$are attained at some $\psi_{ \pm} \in H_{0}^{s}(\Omega)$, respectively. Obviously, $\lambda_{ \pm}>0$, and both $\left|\psi_{ \pm}\right|$are also a minimizer. The same argument as that of proposition 9 of [23] implies that $\psi_{ \pm}$do not change sign in $\Omega$. Thus, $\lambda_{+}$and $-\lambda_{-}$are two eigenvalues of (2.4) with positive eigenfunctions. That is, $\mu\left(\lambda_{+}\right)=\mu\left(-\lambda_{-}\right)=0$. As $\mu(\lambda)$ has only two $\lambda$-intercepts at $\lambda_{s}^{-}(a)$ and $\lambda_{s}^{+}(a)$, we readily infer that $\lambda_{s}^{-}(a)=-\lambda_{-}$and $\lambda_{s}^{+}(a)=\lambda_{+}$. The proof is completed.

Note that the same argument can be easily adopted to give a similar variational characterization for the principal eigenvalues of (2.4) provided that $\mu\left(\lambda^{*}\right)>0$. As a matter of fact, we have $\lambda_{s}^{+}(a)=\lambda^{*}+\lambda_{+}$and $\lambda_{s}^{-}(a)=\lambda^{*}-\lambda_{-}$, where $\lambda_{ \pm}=\inf _{\left\{u \in H_{0}^{s}(\Omega) \mid I_{ \pm}(u)=1\right\}} J_{\lambda^{*}} u$, and

$$
J_{\lambda^{*}}(u)=\frac{c_{n, s}}{2} \int_{\mathbb{R}^{n}} \int_{\mathbb{R}^{n}} \frac{|u(x)-u(y)|^{2}}{|x-y|^{n+2 s}} d y d x+\int_{\Omega}\left[c(x)-\lambda^{*} a(x)\right] u^{2} d x .
$$

We next consider the asymptotic behavior of $\mu(\lambda)$ as $\lambda \rightarrow \infty$ under the conditions that $a(x) \lesseqgtr 0$ in $\Omega$ and that $a(x)$ vanishes in a sub-domain $\Omega_{0}$ of $\Omega$.

Theorem 2.6. Let $\mu(\lambda)$ be the principal eigenvalue of (2.4). Suppose that $c \in C^{\alpha}(\bar{\Omega})$ with some $\alpha \in(0,1)$ and $a \in L^{\infty}(\Omega)$ with $a \lesseqgtr 0$ in $\Omega$. Assume that $\Omega_{0}:=$ int $a^{-1}(0)$ is connected and of class $\mathcal{C}^{2}$ and $\bar{\Omega}_{0} \subset \Omega$. Moreover, $\inf _{\bar{\Omega} \backslash \Omega_{\delta}} a(x)<0$ for sufficiently small $\delta>0$, where $\Omega_{\delta}:=\left\{x \in \bar{\Omega} \mid \operatorname{dist}\left(x, \Omega_{0}\right)<\delta\right\}$. Then $\lim _{\lambda \rightarrow \infty} \mu(\lambda)=\mu_{\Omega_{0}}$, where $\mu_{\Omega_{0}}$ is the principal eigenvalue of $\left\{L_{c}, \mathcal{D}, \Omega_{0}\right\}$.

Proof. Notice that $\mu(\lambda)<\mu_{\Omega_{0}}$. Thus, suffices it to show that for each $\epsilon>0$, there exists $\lambda_{\epsilon}>0$ for which $\mu(\lambda) \geq \mu_{\Omega_{0}}-\epsilon$ provided that $\lambda \geq \lambda_{\epsilon}$. In view of Theorem 2.1, this can be done by showing that $\left\{L_{c}-\lambda a-\left(\mu_{\Omega_{0}}-\epsilon\right), \mathcal{D}, \Omega\right\}$ has a strict super-solution. To obtain a strict supersolution, we first observe that $\lim _{\delta \rightarrow 0} \mu_{\Omega_{\delta}}=\mu_{\Omega_{0}}$, which is guaranteed by Theorem 2.2. Here $\mu_{\Omega_{\delta}}$ is the principal eigenvalue of $\left\{L_{c}, \mathcal{D}, \Omega_{\delta}\right\}$. Therefore, $\mu_{\Omega_{\delta}}>\mu_{\Omega_{0}}-\epsilon$ as long as $\delta$ is sufficiently 
small. Given that $\epsilon>0$, we chose $\delta>0$ such that $\mu_{\Omega_{\delta}} \geq \mu_{\Omega_{0}}-\frac{\epsilon}{2}$. Fix $R>0$, and let $u$ be the unique solution to the following boundary value problem

$$
\left\{\begin{array}{l}
\left(L_{c}-\mu_{\Omega_{0}}+\epsilon\right) u=1-c(x) R+\left(\mu_{\Omega_{0}}-\epsilon\right) R, \quad x \in \Omega_{\delta}, \\
u=0, \quad x \in \mathbb{R}^{n} \backslash \Omega_{\delta} .
\end{array}\right.
$$

Then Remark 1 implies that $\|u\|_{L^{2}\left(\Omega_{\delta}\right)} \leq \epsilon^{-1} 2\|\tilde{f}\|_{L^{2}\left(\Omega_{\delta}\right)}$, where $\tilde{f}=1-c(x) R+\left(\mu_{\Omega_{0}}-\epsilon\right) R$. Hence Proposition 2.1 and Proposition 1.1 of [21] imply that $\|u\|_{C^{s}\left(\mathbb{R}^{n}\right)} \leq \epsilon^{-1} K$, where $K>$ 0 is a constant depending on $n, s, R, c, \mu_{\Omega_{0}}$, and $\Omega_{0}$ only. Note that $(-\Delta)^{s} u=\left(\mu_{\Omega_{0}}-\epsilon-\right.$ $c) u+\tilde{f}$ in $\Omega_{\delta}$ and $u=0$ in $\mathbb{R}^{n} \backslash \Omega_{\delta}$. Let $d(x)=d\left(x, \partial \Omega_{\delta}\right)$. As shown in [22], there holds

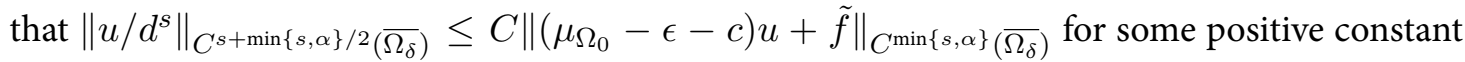
$C$ depending on $n, s$, and $\Omega$. Let $w=u / d^{s}$ in $\Omega_{\delta}$, and $\bar{u}=u+R$. Clearly, we have that $\left(L_{c}-\mu_{\Omega_{0}}+\epsilon\right) \bar{u}=1$ in $\Omega_{\delta}$, and $\bar{u}=R$ in $\mathbb{R}^{n} \backslash \Omega_{\delta}$. In particular, Theorem 2.1 implies that $\bar{u}>0$ in $\Omega_{\delta}$. We next show that $\bar{u}$ is a desired strict supersolution of $\left\{L_{c}-\lambda a-\left(\mu_{\Omega_{0}}-\epsilon\right), \mathcal{D}, \Omega\right\}$ if $\lambda>0$ is sufficiently large. To this end, we first show that $(-\Delta)^{s} \bar{u}$ is bounded in $\mathbb{R}^{n} \backslash \Omega_{\delta}$. Given $x \in \mathbb{R}^{n} \backslash \Omega_{\delta}$, if $x \notin \partial \Omega_{\delta}$, then let $x^{\prime}$ be the project of $x$ on $\partial \Omega_{\delta}$. Then, for every $\varepsilon>0$, we have

$$
\begin{aligned}
\int_{B_{\varepsilon}^{c}(x)} & \frac{\bar{u}(x)-\bar{u}(y)}{|x-y|^{n+2 s}} d y \\
& =\int_{B_{\varepsilon}^{c}(x) \cap \Omega_{\delta}} \frac{\bar{u}(x)-\bar{u}(y)}{|x-y|^{n+2 s}} d y \\
& =\int_{B_{\varepsilon}^{c}(x) \cap \Omega_{\delta}} \frac{d^{s}\left(x^{\prime}\right) w\left(x^{\prime}\right)-d^{s}(y) w(y)}{|x-y|^{n+2 s}} d y \\
& =w\left(x^{\prime}\right) \int_{B_{\varepsilon}^{c}(x) \cap \Omega_{\delta}} \frac{d^{s}\left(x^{\prime}\right)-d^{s}(y)}{|x-y|^{n+2 s}} d y-\int_{B_{\varepsilon}^{c}(x) \cap \Omega_{\delta}} \frac{\left[d^{s}\left(x^{\prime}\right)-d^{s}(y)\right]\left[w\left(x^{\prime}\right)-w(y)\right]}{|x-y|^{n+2 s}} d y \\
& =I_{1, \varepsilon}(x)-I_{2, \varepsilon}(x) .
\end{aligned}
$$

Here $B_{\varepsilon}^{c}(x)=\mathbb{R}^{n} \backslash B_{\varepsilon}(x)$ and we used the fact that

$$
\begin{aligned}
d^{s}(z) w(z)- & d^{s}(y) w(y)= \\
& d^{s}(z)[w(z)-w(y)]+w(z)\left[d^{s}(z)-d^{s}(y)\right]-\left[d^{s}(z)-d^{s}(y)\right][w(z)-w(y)]
\end{aligned}
$$

for $z, y \in \mathbb{R}^{n}$ (see [21]). Lemma 3.1 of [21] shows that $(-\Delta)^{s} d^{s} \in C^{\beta}\left(\overline{\Omega_{\delta, \rho_{0}}}\right)$ for some $\beta \in(0,1)$, where $\Omega_{\delta, \rho_{0}}=\Omega_{\delta} \cap\left\{d<\rho_{0}\right\}$, and $\rho_{0}>0$ depends on $\Omega_{\delta}$ only. In fact, a careful look into the proof of Lemma 3.1 of [21] shows that $\rho_{0}$ can be so chosen that it is independent of $\delta$ if $\delta$ is sufficiently small. Since $|x-y| \geq\left|x^{\prime}-y\right|$, and $w \in C^{s+\min \{s, \alpha\} / 2}\left(\overline{\Omega_{\delta}}\right)$, it follows that $\left|I_{1, \varepsilon}\right|$ are uniformly bounded for $x \in \Omega \backslash \Omega_{\delta}$ and $\varepsilon>0$. Additionally, as $\left|d^{s}\left(x^{\prime}\right)-d^{s}(y)\right|\left|w\left(x^{\prime}\right)-w(y)\right| \leq$ $\epsilon^{-1} C\left|x^{\prime}-y\right|^{2 s+\gamma}$, where $\gamma=\min \{1-s, \min \{s, \alpha\} / 2\}$, and $C$ is the constant specified above, we have $\left|d^{s}\left(x^{\prime}\right)-d^{s}(y) \| w\left(x^{\prime}\right)-w(y)\right|\left|x^{\prime}-y\right|^{-(n+2 s)} \in L^{1}\left(\Omega_{\delta}\right)$, and so $\left|I_{2, \varepsilon}\right|$ are uniformly bounded 
as well. Hence, $\left|(-\Delta)^{s} \bar{u}\right|$ is bounded in $x \in \Omega \backslash \Omega_{\delta}$, and $\left|(-\Delta)^{s} \bar{u}\right| \leq \epsilon^{-1} C$, where $C>0$ is a constant depending on $n, s, R, c, \mu_{\Omega_{0}}, \Omega_{0}$, and $\Omega$. Consequently, $\left(L_{c}-\left(\mu_{\Omega_{0}}-\epsilon\right) \bar{u} \in L^{\infty}(\Omega)\right.$. Since inf ${\overline{\bar{\Omega}} \backslash \Omega_{\delta}} a<0$, there exists $\lambda_{\epsilon}$ such that $\left(L_{c}-\lambda a-\mu_{\Omega_{0}}-\epsilon\right) \bar{u} \geq 0$ in $\Omega$ provided that $\lambda \geq \lambda_{\epsilon}$. It then follows from Theorem 2.1 that $\mu(\lambda)>\mu_{\Omega_{0}}-\epsilon$ if $\lambda \geq \lambda_{\epsilon}$. The proof is completed.

We now conclude section 2 with a brief discussion on an optimization problem for positive principal eigenvalues of (2.4). Our discussion is motivated by [8] and provides a answer for the minimization of positive principal eigenvalues of (2.4), which is parallel to Theorem 3.9 of [8].

Theorem 2.7. Let $\Omega \subset \mathbb{R}^{n}$ be a bounded domain with Lipschitz boundary. Let $\mu(0)$ be the principal eigenvalue of $\left\{L_{c}, \mathcal{D}, \Omega\right\}$. Let $\mathcal{M}=\left\{a(x) \in L^{\infty}(\Omega) \mid-a_{2} \leq a(x) \leq a_{2} ; \bar{a}=a_{0} ; a(x) \gtrless\right.$ 0 in $\left.\Omega_{a}\right\}$, where $a_{0}, a_{1}$, and $a_{2}$ are constants with $a_{1}, a_{2}>0,-a_{2}|\Omega|<a_{0} \leq a_{1}|\Omega|, \Omega_{a} \subset \Omega$ is a nonempty open subset, and $\bar{a}=|\Omega|^{-1} \int_{\Omega} a d x$. Let $\lambda_{s}^{+}(a)$ be the positive principal eigenvalue of (2.4). Suppose that $\mu(0)>0$. Then there exist a measurable set $E \subseteq \Omega$ and a simple function $a^{*}=a_{1} \chi_{E}-a_{2} \chi_{\Omega \backslash E} \in \mathcal{M}$ such that $\lambda_{s}^{+}\left(a^{*}\right)=\inf _{a \in \mathcal{M}} \lambda_{s}^{+}(a)$.

Proof. The proof is similar to that for Theorem 3.9 of [8]. Only part of it needs elaboration for the sake of clarity. As shown in [8], for each $w \in L^{2}(\Omega)$, there exists a measurable set $E_{w} \subset \Omega$ such that $a_{1} \chi_{E_{w}}-a_{2} \chi_{E_{w}^{c}}$ solves $\sup _{a \in \mathcal{M}} \int_{\Omega} a w^{2} d x$. Let $w \in L^{2}(\Omega)$, we write $a_{w}^{*}=a_{1} \chi_{E_{w}}-a_{2} \chi_{E_{w}^{c}}$. Given that $a \in \mathcal{M}$, let $\varphi$ be the eigenfunction for $\lambda_{s}^{+}(a)$. Note that $\int a_{\varphi}^{*} \varphi^{2} d x \geq \int a \varphi^{2} d x$. Hence, we have

$$
\lambda_{s}^{+}\left(a_{\varphi}^{*}\right)=\inf _{u \in H_{0}^{s}, \int a u^{2} d x>0} \frac{J(u)}{\int_{\Omega} a_{\varphi}^{*} u^{2} d x} \leq \frac{J(\varphi)}{\int_{\Omega} a_{\varphi}^{*} \varphi^{2} d x} \leq \frac{J(\varphi)}{\int_{\Omega} a \varphi^{2} d x} \leq \lambda_{s}^{+}(a) .
$$

This implies that $\lambda_{s}^{0}=\inf _{a \in \mathcal{M}} \lambda_{s}^{+}(a)=\inf \left\{\lambda_{s}^{+}\left(a_{1} \chi_{E}-a_{2} \chi_{\Omega \backslash E}\right)\right\}$. To complete the proof, we only need to show $\lambda_{s}^{0}$ is attainable. Choose a sequence $\left\{a_{n}, \lambda_{s}^{+}\left(a_{n}\right)\right\}$ with $a_{n} \in \mathcal{M}$ such that $\lim _{n \rightarrow \infty} \lambda_{s}^{+}\left(a_{n}\right)=\lambda_{s}^{0}$. Let $\varphi_{n}$ be the positive eigenfunction associated with $\lambda_{s}^{+}\left(a_{n}\right)$. Since $J\left(\varphi_{n}\right)$ are bounded, there exists a subsequence, still denoted by $\left\{\varphi_{n}\right\}$, such that $\varphi_{n}$ weakly converge to $\varphi_{*}$ in $H_{0}^{s}(\Omega)$ for some $\varphi_{*} \in H_{0}^{s}(\Omega)$, and $\lim _{n \rightarrow \infty}\left\|\varphi_{n}-\varphi_{*}\right\|_{L^{2}(\Omega)}=0$. In particular, $\lim _{n \rightarrow \infty} J\left(\varphi_{n}\right)=\lambda_{s}^{0} \geq J\left(\varphi_{*}\right) \geq \mu(0)\left\|\varphi_{*}\right\|_{L^{2}(\Omega)}$. Observe that $\lim _{n \rightarrow \infty} \int_{\Omega} a_{n}\left(\varphi^{2}-\varphi_{*}^{2}\right) d x=0$, and $\int_{\Omega} a_{n} \varphi_{n}^{2} d x=1$. Thus, $\varphi^{*} \neq 0$. Now let $\phi_{n}=\left(J\left(\varphi_{n}\right)\right)^{-\frac{1}{2}} \varphi_{n}$ and $\phi_{*}=\left(J\left(\varphi_{*}\right)\right)^{-\frac{1}{2}} \varphi_{*}$. Then, we have

$$
\begin{aligned}
& \frac{1}{\lambda_{s}^{0}} \geq \frac{1}{\lambda_{s}^{+}\left(a_{\phi_{*}}^{*}\right)} \geq \int_{\Omega} a_{\phi_{*}}^{*} \phi_{*}^{2} d x \geq \int_{\Omega} a_{n} \phi_{*}^{2} d x=\frac{\lambda_{s}^{0}}{J\left(\varphi_{*}\right)} \int_{\Omega} a_{n}\left(\frac{\varphi_{*}}{\sqrt{\lambda_{s}^{0}}}\right)^{2} d x, \\
& \int_{\Omega} a_{n} \phi_{n}^{2} d x=\frac{1}{\lambda_{s}^{+}\left(a_{n}\right)} .
\end{aligned}
$$


Hence

$$
\frac{1}{\lambda_{s}^{0}} \geq \frac{1}{\lambda_{s}^{+}\left(a_{\phi_{*}}^{*}\right)} \geq \int_{\Omega} a_{\phi_{*}}^{*} \phi_{*}^{2} d x \geq \frac{\lambda_{s}^{0}}{J\left(\varphi_{*}\right)}\left\{\int_{\Omega} a_{n} \phi_{n}^{2} d x+\int_{\Omega} a_{n}\left[\left(\frac{\varphi_{*}}{\sqrt{\lambda_{s}^{0}}}\right)^{2}-\phi_{n}^{2}\right] d x\right\} .
$$

By passing the limits as $n \rightarrow \infty$, we find that $\lambda_{s}^{0}=J\left(\varphi_{*}\right)$ and $\lambda_{s}^{0}=\lambda_{s}^{+}\left(a_{\phi_{*}}^{*}\right)$. The proof is completed.

\section{Cooperative systems}

This section is focused on the principal eigenvalues and a maximum principle for cooperative systems involving fractional Laplace operators. The results established in the section are a counterpart of those given in [18] for cooperative elliptic systems. We again assume that $\Omega \subset \mathbb{R}^{n}$ is a bounded domain with smooth boundary and first consider boundary value problem

$$
\left\{\begin{array}{l}
(-\Delta)^{s_{i}} u_{i}+\sum_{j=1}^{k} c_{i, j} u_{j}=f_{i}(x) \quad \text { in } \Omega \quad(1 \leq i \leq k) \\
u_{i}=0 \text { in } \mathbb{R}^{n} \backslash \Omega
\end{array}\right.
$$

where $c_{i, j} \in L^{\infty}(\Omega), 1 \leq i, j \leq k$, and $s_{i} \in(0,1), k \geq 2$ stands for an integer. Throughout this section, we shall assume without loss of generality that $0<s_{1} \leq \cdots \leq s_{i} \leq s_{i+1} \leq \cdots \leq s_{k}<$ 1.

Definition 6. Let $\Omega \subset \mathbb{R}^{n}$ be a bounded domain. Given $f=\left(f_{1}, \cdots, f_{k}\right)$ with $f_{i} \in L^{2}(\Omega)$, a function $w=\left(w_{1}, \cdots, w_{k}\right)$ with $w_{i} \in H_{0}^{s_{i}}(\Omega)$ is said to be a weak solution to the boundary value problem (3.1) if

$$
\frac{c_{n, s_{i}}}{2} \int_{\mathbb{R}^{n}} \int_{\mathbb{R}^{n}} \frac{\left[w_{i}(x)-w_{i}(y)\right]\left[\phi_{i}(x)-\phi_{i}(y)\right]}{|x-y|^{n+2 s}} d y d x+\int_{\Omega} \sum_{j=1}^{k} c_{i, j} w_{j} \phi_{i} d x=\int_{\Omega} f_{i} \phi_{i} d x
$$

for any $\phi=\left(\phi_{1}, \cdots, \phi_{k}\right)$ with $\phi_{i} \in H_{0}^{s_{i}}(\Omega)$ for $i \in\{1, \cdots, k\}$.

Proposition 3.1. Let $\Omega \subset \mathbb{R}^{n}$ be a bounded domain with Lipschitz boundary. Let $w=\left(w_{1}, \cdots, w_{k}\right)$ with $w_{i} \in H_{0}^{s_{i}}(\Omega)(i=1, \cdots, k)$ be a weak solution of (3.1). Namely,

$$
\sum_{i=1}^{k}\left\{\int_{\mathbb{R}^{n}} \int_{\mathbb{R}^{n}} \frac{\left[w_{i}(x)-w_{i}(y)\right]\left[\phi_{i}(x)-\phi_{i}(y)\right]}{|x-y|^{n+2 s_{i}}} d y d x+\sum_{j=1}^{k} \int_{\Omega} c_{i, j} w_{j} \phi_{i} d x\right\}=\sum_{i=1}^{k} \int_{\Omega} f_{i} \phi_{i} d x
$$

for any $\phi=\left(\phi_{1}, \cdots, \phi_{k}\right)$ with $\phi_{i} \in H_{0}^{s_{i}}(\Omega)$. Assume that $c_{i, j} \in L^{\infty}(\Omega)(i \neq j, i, j=1, \cdots, k)$, $c_{i i} \in L^{p}(\Omega)$, and $f_{i} \in L^{p}(\Omega)(i=1, \cdots, k), n>2 s_{k}$, where $p>\max \left\{n / 2 s_{1}, 2 /\left[1-\frac{2\left(s_{k}-s_{1}\right)}{n-2 s_{k}}\right]\right\}$. Then there exists a constant $C>0$ such that

$$
\left\|w_{i}\right\|_{L^{\infty}(\Omega)} \leq C, \quad 1 \leq i \leq k
$$

where $C$ depends only on $|\Omega|, n, p, s_{i},\left\|c_{i, j}\right\|_{L^{\infty}(\Omega)}(i \neq j),\left\|w_{i}\right\|_{L^{2}(\Omega)}\left\|c_{i i}\right\|_{L^{p}(\Omega)}$, and $\left\|f_{i}\right\|_{L^{p}(\Omega)}$. 
Proof. We fix $q_{1}=\frac{p}{p-1}$ and set $q_{i}>1(2 \leq i \leq k)$ such that $\frac{n}{\left(n-2 s_{1}\right) q_{1}}=\frac{n}{\left(n-2 s_{2}\right) q_{2}}=$ $\cdots=\frac{n}{\left(n-2 s_{k}\right) q_{k}}$. Let $\chi=\frac{n}{\left(n-2 s_{1}\right) q_{1}}$. Clearly $\chi>1$, and $2>q_{k} \geq \cdots \geq q_{i} \geq \cdots \geq q_{1}$. The Moser iteration is again utilized to deduce the conclusion. To implement each iteration, we always chose an ordered pair $\left(\beta_{1}, \cdots, \beta_{k}\right)$ so that $\left(1+\beta_{1}\right) q_{1}=\left(1+\beta_{2}\right) q_{2}=\cdots=\left(1+\beta_{k}\right) q_{k}$. Let $w_{i, m}=\min \left\{\left|w_{i}\right|, m\right\}$, where $m>0$ is a given number. Similar to the proof of Proposition 2.1, Multiplying the $i$ th equation of (3.1) by $\left(w_{i, m}+1\right)^{\beta_{i}}-1$ and integrating the resulting equation over $\Omega$ give

$$
\begin{aligned}
& \left(\int_{\Omega}\left(\left|w_{i, m}\right|+1\right)^{\frac{\left(1+\beta_{i}\right) n}{n-2 s_{i}}} d x\right)^{\frac{n-2 s_{i}}{n}} \leq C\left(n, s_{i}\right)\left(\frac{\beta_{i}+1}{2 \sqrt{\beta_{i}}}\right)^{2} \sum_{j=1}^{k} \int_{\Omega}\left|c_{i, j}\right|\left|w_{j}\right|\left(\left|w_{i, m}\right|+1\right)^{\beta_{i}} d x \\
& +C\left(n, s_{i}\right)\left(\frac{\beta_{i}+1}{2 \sqrt{\beta_{i}}}\right)^{2}\left\{\int_{\Omega}\left|f_{i}\right|\left(\left|w_{i, m}\right|+1\right)^{\beta_{i}} d x+|\Omega|^{\frac{n-2 s_{i}}{n}-\frac{1}{q_{i}}} \int_{\Omega}\left(\left|w_{i, m}\right|+1\right)^{\left(1+\beta_{i}\right) q_{i}} d x\right\} .
\end{aligned}
$$

Let $r_{i}=\frac{\left(1+\beta_{i}\right) q_{i}}{\beta_{i}}$ and $t_{i}=\frac{\left(1+\beta_{i}\right) q_{i}}{\left(1+\beta_{i}\right) q_{i}-\beta_{i}}$. For $j \neq i$, using Hölder's inequality and Cauchy inequality yield that

$$
\begin{aligned}
& \int_{\Omega}\left|c_{i, j}\right|\left|w_{j}\right|\left(\left|w_{i, m}\right|+1\right)^{\beta_{i}} d x \leq \sup \left|c_{i, j}\right||| w_{j}\left\|_{L^{t_{i}(\Omega)}}\right\|\left(\left|w_{i, m}\right|+1\right)^{\beta_{i}} \|_{L^{r_{i}(\Omega)}} \\
& \leq \sup \left|c_{i, j}\right| \frac{1}{\beta_{i}+1}\left(\int_{\Omega}\left|w_{j}\right|^{\frac{\left(1+\beta_{i}\right) q_{i}}{\left(1+\beta_{i}\right) q_{i}-\beta_{i}}} d x\right)^{\frac{\left(1+\beta_{i}\right) q_{i}-\beta_{i}}{q_{i}}}+\frac{\beta_{i}}{\beta_{i}+1}\left(\int_{\Omega}\left(\left|w_{i, m}\right|+1\right)^{\left(1+\beta_{i}\right) q_{i}} d x\right)^{\frac{1}{q_{i}}} \\
& \leq\left.\sup \left|c_{i, j}\right| \Omega\right|^{\frac{\left(1+\beta_{i}\right)\left(q_{i}-1\right)}{q_{i}}}\left(\int_{\Omega}\left|w_{j}\right|^{\left(1+\beta_{i}\right) q_{i}} d x\right)^{\frac{1}{q_{i}}}+\left(\int_{\Omega}\left(\left|w_{i, m}\right|+1\right)^{\left(1+\beta_{i}\right) q_{i}} d x\right)^{\frac{1}{q_{i}}} .
\end{aligned}
$$

Let $p_{i}=\frac{q_{i}}{q_{i}-1}$. Note that $p_{i} \leq p$. Then, we find that

$$
\begin{aligned}
\left(\int _ { \Omega } \left(\left|w_{i, m}\right|\right.\right. & \left.+1)^{\frac{\left(1+\beta_{i}\right) n}{n-2 s_{i}}} d x\right)^{\frac{n-2 s_{i}}{n}} \\
& \leq C\left(n, s_{i}\right)\left(\frac{1+\beta_{i}}{2 \sqrt{\beta_{i}}}\right)^{2}\left\{\left\|c_{i i}\right\|_{L^{p_{i}(\Omega)}}\left(\int_{\Omega}\left[\left(\left|w_{i}\right|+1\right)\left(\left|w_{i, m}\right|+1\right)^{\beta_{i}}\right]^{q_{i}} d x\right)^{\frac{1}{q_{i}}}\right. \\
& +\sup _{i \neq j}\left|c_{i, j}\right||\Omega|^{\frac{\left(1+\beta_{i}\right)\left(q_{i}-1\right)}{q_{i}}} \sum_{j=1, j \neq i}^{k}\left(\int_{\Omega}\left(\left|w_{j}\right|+1\right)^{\left(1+\beta_{i}\right) q_{i}} d x\right)^{\frac{1}{q_{i}}} \\
& \left.+\left[k-1+|\Omega|^{\frac{n-2 s_{i}}{n}-\frac{1}{q_{i}}}+\left\|f_{i}\right\|_{L^{p_{i}(\Omega)}}\right]\left(\int_{\Omega}\left(\left|w_{i, m}\right|+1\right)^{\left(1+\beta_{i}\right) q_{i}} d x\right)^{\frac{1}{q_{i}}}\right\} .
\end{aligned}
$$

Let $\gamma_{q_{k}}>0$ be the number such that $\frac{1+\beta}{2 \sqrt{\beta}} \leq \sqrt{\gamma_{q_{k}}(1+\beta)}$ provided that $\beta \geq \frac{2-q_{k}}{q_{k}}$. Write $C_{i}^{\prime}=$ $C\left(n, s_{i}\right) \gamma_{q_{k}}\left(1+\beta_{i}\right)\left[\sup _{i \neq j}\left|c_{i, j}\right||\Omega|^{\frac{\left(1+\beta_{i}\right)\left(q_{i}-1\right)}{q_{i}}}+k-1+|\Omega|^{\frac{n-2 s_{i}}{n}-\frac{1}{q_{i}}}+\left\|c_{i i}\right\|_{L^{p_{i}(\Omega)}}+\left\|f_{i}\right\|_{L^{p_{i}}(\Omega)}\right]$. Note that $\beta_{i} \geq \frac{2-q_{k}}{q_{k}}$ for all $i$ whenever $\beta_{k} \geq \frac{2-q_{k}}{q_{k}}$. By passing the limits as $m \rightarrow \infty$, we have

$$
\left(\int_{\Omega}\left(\left|w_{i}\right|+1\right)^{\frac{\left(1+\beta_{i}\right) n}{n-2 s_{i}}} d x\right)^{\frac{n-2 s_{i}}{n}} \leq C_{i}^{\prime}\left(\sum_{j=1}^{k} \int_{\Omega}\left(\left|w_{j}\right|+1\right)^{\left(1+\beta_{i}\right) q_{i}} d x\right)^{\frac{1}{q_{i}}} .
$$


Let again $\theta=\left(1+\beta_{i}\right) q_{i}$ and $\chi=\frac{n}{n-2 s_{i}} \frac{1}{q_{i}}$, and assume without loss of generality that $|\Omega|>1$, then

$$
\left(\int_{\Omega}\left(\left|w_{i}\right|+1\right)^{\chi \theta} d x\right)^{\frac{1}{\chi \theta}} \leq\left(\frac{c_{1} \theta}{q_{1}}\right)^{\frac{q_{k}}{\theta}}\left[|\Omega| c_{3}^{\frac{q_{k}}{\theta}}+c_{2}^{\frac{q_{k}}{\theta}}\right]\left(\sum_{j=1}^{k} \int_{\Omega}\left(\left|w_{j}\right|+1\right)^{\theta} d x\right)^{\frac{1}{\theta}}
$$

where $c_{1}=\max _{1 \leq i \leq k} C\left(n, s_{i}\right) \gamma_{q_{k}}, c_{2}=k-1+|\Omega|^{\frac{n-2 s_{1}}{n}}+\max _{1 \leq i \leq k}\left(\left\|c_{i i}\right\|_{L^{p_{i}(\Omega)}}+\left\|f_{i}\right\|_{L^{p_{i}(\Omega)}}\right)$, and $c_{3}=\sup _{i \neq j}\left|c_{i, j}\right|_{L^{\infty}}$. In view of the fact that $\left(\sum_{i=1}^{k}\left|a_{i}\right|\right)^{t} \leq \sum_{i=1}^{k}\left|a_{i}\right|^{t}$ for any $0<t<1$ and $a_{i} \in \mathbb{R}$, we have

$$
\left(\sum_{i=1}^{k} \int_{\Omega}\left(\left|w_{i}\right|+1\right)^{\chi \theta} d x\right)^{\frac{1}{\chi^{\theta}}} \leq k\left(\frac{c_{1} \theta}{q_{1}}\right)^{\frac{q_{k}}{\theta}}\left[|\Omega| c_{3}^{\frac{q_{k}}{\theta}}+c_{2}^{\frac{q_{k}}{\theta}}\right]\left(\sum_{i=1}^{k} \int_{\Omega}\left(\left|w_{i}\right|+1\right)^{\theta} d x\right)^{\frac{1}{\theta}} .
$$

Set $\theta_{m+1}=\chi \theta_{m}$ and $\theta_{0}=2$. Then by induction, we have

$$
\left\|\left(\left|w_{i}\right|+1\right)\right\|_{L^{\theta_{m+1}(\Omega)}} \leq \prod_{j=0}^{m} k\left(\frac{c_{1} \theta_{j}}{q_{1}}\right)^{\frac{q_{k}}{\theta_{j}}}\left[|\Omega| c_{3}^{\frac{q_{k}}{\theta}}+c_{2}^{\frac{q_{k}}{\theta_{j}}}\right]\left(\sum_{j=1}^{k}\left\|\left(\left|w_{j}\right|+1\right)\right\|_{L^{2}(\Omega)}^{2}\right)^{\frac{1}{2}} .
$$

Since $\chi>1$, there exists $m$ such that $\theta_{m+1}>p$. Namely, after finite number of iterations, we find that $w_{i} \in L^{p}(\Omega)(1 \leq i \leq k)$. Write that $\widehat{f}_{i}=f_{i}+\sum_{j=1, j \neq i}^{k} c_{i, j} w_{j}$, clearly, $\widehat{f}_{i} \in L^{p}(\Omega)$. Thus, Proposition 2.1 implies that $w_{i} \in L^{\infty}(\Omega)(1 \leq i \leq k)$. The proof is completed.

Lemma 3.1. Let $\Omega \subset \mathbb{R}^{n}$ be a bounded domain with Lipschitz boundary. Suppose that $c_{i, j} \in$ $L^{\infty}(\Omega)(1 \leq i, j \leq k)$. Given $f_{i} \in L^{2}(\Omega)(1 \leq i \leq k)$, then the boundary value problem

$$
\left\{\begin{array}{l}
(-\Delta)^{s_{i}} u_{i}+\mu u_{i}+\sum_{j=1}^{m} c_{i, j} u_{j}=f_{i} \quad \text { in } \Omega \\
u_{i}=0 \text { in } \mathbb{R}^{n} \backslash \Omega
\end{array}\right.
$$

has a unique weak solution $w=\left(w_{1}, \cdots, w_{k}\right)$ with $w_{i} \in H_{0}^{s_{i}}(\Omega)$ provided that $\mu \in \mathbb{R}$ is suffciently large. In particular, if $c_{i, j} \leq 0$ whenever $i \neq j$ and $f_{i} \geq 0$ for each $i$, then $w_{i} \geq 0$.

Proof. The existence and uniqueness of a weak solution shall be established via the Lax-Milgram theorem. To this end, we set

$$
\begin{aligned}
& X=\prod_{j=1}^{k} H_{0}^{s_{j}}(\Omega), \\
& Y=\underbrace{L^{2}(\Omega) \times \cdots \times L^{2}(\Omega)}_{k} .
\end{aligned}
$$

For $u=\left(u_{1}, \cdots, u_{k}\right)$, and $v=\left(v_{1}, \cdots, v_{k}\right) \in Y$ or $X$, define

$$
(u \mid v)=\sum_{j=1}^{k} \int_{\Omega} u_{j} v_{j} d x
$$




$$
\langle u \mid v\rangle=\sum_{j=1}^{k}\left[\int_{\mathbb{R}^{2 n}} \frac{\left[u_{j}(x)-u_{j}(y)\right]\left[v_{j}(x)-v_{j}(y)\right]}{|x-y|^{n+2 s_{i}}} d y d x+\int_{\Omega} u_{j} v_{j} d x\right],
$$

and $\|u\|_{X}=\sqrt{\langle u \mid u\rangle},\|u\|_{Y}=\sqrt{(u \mid u)}$. Clearly, both $X$ and $Y$ are Hilbert spaces. Now Let $a(u, v): X \times X \rightarrow \mathbb{R}$ be defined by

$$
\begin{gathered}
a(u, v)=\sum_{i=1}^{k}\left\{\frac{c_{n, s_{i}}}{2} \int_{\mathbb{R}^{n}} \int_{\mathbb{R}^{n}} \frac{\left[u_{i}(x)-u_{i}(y)\right]\left[v_{i}(x)-v_{i}(y)\right]}{|x-y|^{n+2 s_{i}}} d y d x+\right. \\
\left.\sum_{j=1}^{k} \int_{\Omega} c_{i, j} u_{j} v_{i} d x\right\} .
\end{gathered}
$$

It follows from Sobolve embedding inequality and Hölder inequality that

$$
|a(u, v)+\mu(u \mid v)| \leq C_{\mu, 1}\|u\|_{X}\|v\|_{X}, \quad a(u, u)+\mu(u \mid u) \geq C_{\mu, 2}\|u\|_{X}^{2}
$$

for some constants $C_{\mu, 1}>0$ and $C_{\mu, 2}$. It is also easy to see that $C_{\mu, 2} \geq 0$ provided that $\mu$ is sufficiently large. Following [16], we let $\mathcal{L}_{c}: D\left(\mathcal{L}_{c}\right) \rightarrow Y$ be the unbounded linear operator associated with $a(\cdot, \cdot)$ with $a(u, v)=\left(\mathcal{L}_{c} u \mid v\right)$ for $u, v \in X$. Here

$D\left(\mathcal{L}_{c}\right)=\{u \in X \mid v \longmapsto a(u, v)$ is continuous on $X$ in terms of the topology induced by $Y\}$.

Similarly, we let $\mathcal{L}_{c}+\mu I$ be the linear operator associated with $a(u, v)+\mu(u \mid v)$. Notice that $D\left(\mathcal{L}_{c}\right)$ is dense in $X$. As a matter of fact, $\underbrace{C_{0}^{2}(\Omega) \times \cdots \times C_{0}^{2}(\Omega)}_{k} \subset D\left(\mathcal{L}_{c}\right)$. Hence Theorem 3.6 of [16] implies that $\left(\mathcal{L}_{c}+\mu I\right)$ has bounded inverse as $\mu$ is sufficiently large. In other words, the boundary value problem (3.2) has a unique weak solution $\left(\mathcal{L}_{c}+\mu I\right)^{-1} f$, where $f \in Y$. Note that $\mathcal{L}_{c}$ is Fredholm due to the compactness of $\left(\mathcal{L}_{c}+\mu I\right)^{-1}$ since $X$ is compactly embedded in $Y$. We now proceed to complete the last part of this lemma. Assume that $c_{i, j} \leq 0$ if $i \neq j$. Let $\phi_{i}=w_{i}^{-}$, where $w_{i}^{-}=\min \left\{w_{i}, 0\right\}$. Then

$$
\begin{gathered}
\sum_{i=1}^{k}\left\{\frac{c_{n, s_{i}}}{2} \int_{\mathbb{R}^{2 n}} \frac{\left[w_{i}^{-}(x)-w_{i}^{-}(y)\right]^{2}+2 w_{i}^{+}(x) w_{i}^{-}(y)}{|x-y|^{n+2 s_{i}}} d y d x+\right. \\
\left.\mu \int_{\Omega}\left|w_{i}^{-}\right|^{2} d x-\sum_{j=1, j \neq i}^{k} \int_{\Omega} c_{i, j} w_{j}^{+} w_{i}^{-} d x\right\} \\
=-\sum_{i=1}^{k}\left\{\sum_{j=1}^{k} \int_{\Omega} c_{i, j} w_{j}^{-} w_{i}^{-} d x+\int_{\Omega} f_{i} w_{i}^{-} d x\right\} .
\end{gathered}
$$

In view of the fact that $c_{i, j} \leq 0$ if $i \neq j$, and using the Hölder inequality, we find 


$$
\begin{aligned}
\sum_{i=1}^{k}\left\{\frac{c_{n, s_{i}}}{2}\right. & \left.\int_{\mathbb{R}^{2 n}} \frac{\left[w_{i}^{-}(x)-w_{i}^{-}(y)\right]^{2}}{|x-y|^{n+2 s_{i}}} d y d x+\mu \int_{\Omega}\left|w_{i}^{-}\right|^{2} d x\right\} \\
& \leq \sum_{i=1}^{k}\left\{\sum_{j=1}^{k} \int_{\Omega}\left|c_{i, j}\right|\left|w_{j}^{-} w_{i}^{-}\right| d x-\int_{\Omega} f_{i} w_{i}^{-} d x\right\} \\
& \leq 2 \max _{1 \leq i, j \leq k}\left|c_{i, j}\right| L^{\infty} \sum_{j=1}^{k} \int_{\Omega}\left|w_{j}^{-}\right|^{2} d x-\sum_{j=1}^{k} \int_{\Omega} f_{i} w_{i}^{-} d x .
\end{aligned}
$$

Let $\lambda_{\text {min }}=\min _{1 \leq i \leq k}\left\{\lambda_{s_{i}}\right\}$, where $\lambda_{s_{i}}$ is the principal eigenvalue of $(-\Delta)^{s_{i}}$ subject to Dirichlet boundary condition in $\Omega$, then as long as $\mu>2 \max _{1 \leq i, j \leq k}\left|c_{i, j}\right|_{L^{\infty}}-\lambda_{\text {min }}$, we have

$$
\theta \sum_{i=1}^{k} \int_{\Omega}\left|w_{i}^{-}\right|^{2} d x \leq-\sum_{j=1}^{k} \int_{\Omega} f_{i} w_{i}^{-} d x
$$

Here $\theta=\mu-2 \max _{1 \leq i, j \leq k}\left|c_{i, j}\right|_{L^{\infty}}+\lambda_{\min }$. It immediately follows that $w_{i}^{-}=0(1 \leq i \leq k)$. The proof is completed.

Theorem 3.1. Let $\mathcal{L}_{c}$ be defined in Lemma 3.1. Suppose that $\Omega \subset \mathbb{R}^{n}$ is a bounded domain with $\partial \Omega \in \mathcal{C}^{1}$, and $n>2 s_{k}$. Assume that $c_{i, j} \in L^{\infty}(\Omega)$ and $c_{i, j} \leq 0$ if $i \neq j$. Moreover, there exists a permutation $p_{1} p_{2} \cdots p_{k}$ of $\{1, \cdots, k\}$ for which $c_{p_{i} p_{i+1}} \lesseqgtr 0$ in $\Omega(i=1, \cdots, k-1)$, and $c_{p_{k} p_{1}} \lesseqgtr 0$ in $\Omega$. Then $\mathcal{L}_{c}$ possesses exactly one real eigenvalue $\lambda_{\mathbf{s}}$ that is equal to $\mathfrak{s}\left(\mathcal{L}_{c}\right)$, where $\mathfrak{s}\left(\mathcal{L}_{c}\right)=\inf \left\{\Re \lambda \mid \lambda \in \sigma\left(\mathcal{L}_{c}\right)\right\}$. In particular, $\lambda_{\mathbf{s}}$ is a simple eigenvalue. Assume further that $c_{i, j}=c_{j, i}$ provided that $i \neq j$, then $\lambda_{\mathbf{s}}=\inf _{u \in X,\|u\|_{X}=1} a(u, u)$, where $a(u, v)$ is defined by (3.3).

Proof. As shown in the proof of Lemma 3.1, $\left(\mathcal{L}_{c}+\mu I\right)^{-1}$ is positive if $\mu$ is sufficiently large. Let $r_{\mu}$ be the spectral radius of $\left(\mathcal{L}_{c}+\mu I\right)^{-1}$. To show that $r_{\mu}>0$ provided that $\mu$ is sufficiently large, let $\Omega^{\prime} \subset \Theta \subset \Omega$ be a bounded domain, where $\Theta$ is a compact subset of $\Omega$. Let $f=\left(f_{1}, \cdots, f_{k}\right)$ be so chosen that $f_{i} \gtrless 0$ and $f_{i} \in C_{c}\left(\Omega^{\prime}\right)$. Write $\psi=\left(\psi_{1}, \cdots, \psi_{k}\right)=\left(\mathcal{L}_{c}+\mu I\right)^{-1} f$. Thus, Lemma 3.1 shows that $\psi_{k} \in H_{0}^{s_{i}}(\Omega)$ and $\psi_{k} \geq 0$. Moreover, Proposition 3.1 and Theorem 1.2 of [21] imply that $\psi_{k} \in C^{s_{i}}\left(\mathbb{R}^{n}\right)$. Note that $(-\Delta)^{s_{i}} \psi_{k}+c_{i i} \psi_{k} \geq 0$ in $\Omega$, and $\psi_{k}=0$ in $\Omega^{c}$, thus, it follows from the Proposition 2.3 that either $\psi_{i}>0$ or $\psi_{i}=0$ in $\Omega$ for each $i$. Due to the assumption, if $\psi_{i}=0$ for some $i$, then $\psi_{i}=0$ for all $i$. As $f_{i} \gtrless 0$, we see that $\psi_{i}>0$ in $\Omega$ for all $i$. Hence there exists $\theta>$ 0 such that $\psi \geq \theta f$, that is, $\left(\mathcal{L}_{c}+\mu I\right)^{-1} f \geq \theta f$, and hence $\left.\sigma\left(\mathcal{L}_{c}+\mu I\right)^{-1}\right) \backslash\{0\} \neq \varnothing$. Then the Krein-Rutman theorem implies that $1 / r_{\mu}-\mu$ is an eigenvalue of $\mathcal{L}_{c}$ with a positive eigenfunction $\left(\phi_{1}, \cdots, \phi_{k}\right) \geq 0$. Write $\lambda_{s}=1 / r_{\mu}-\mu$. It follows from the same reasoning as above that $\phi_{i} \in C^{s_{i}}\left(\mathbb{R}^{n}\right)$, and $(-\Delta)^{s_{i}} \phi_{i}+\sum_{i=1}^{k} c_{i, j} \phi_{j}=\lambda_{\mathbf{s}} \phi_{i}$ in $\Omega$, and $\phi_{i}=0$ in $\Omega^{c}$. In particular, $\phi_{i}>0$ in $\Omega$ for all $i$. Meanwhile, as $\mathcal{L}_{c}$ is resolvent positive, $\mathfrak{s}\left(\mathcal{L}_{c}\right)$ is also an eigenvalue of $\mathcal{L}_{c}$. Obviously, 
$\mathfrak{s}\left(\mathcal{L}_{c}\right) \leq \lambda_{\mathbf{s}}$. Next we show that $\mathfrak{s}\left(\mathcal{L}_{c}\right)=\lambda_{\boldsymbol{s}}$. To this end, we let $\left(\phi_{1}, \cdots, \phi_{k}\right) \in \operatorname{ker}\left(\mathfrak{s}\left(\mathcal{L}_{c}\right) I-\mathcal{L}_{c}\right)$, and assume without loss of generality that $\varphi_{1}\left(x_{1}\right)<0$ for some $x_{1} \in \Omega$. In view of Theorem 1.2 of [21], there exists $C>0$ such that $\left|\varphi_{i}(x)\right| \leq C d(x, \partial \Omega)^{s_{i}}$. Now assume to the contrary that $\mathfrak{s}\left(\mathcal{L}_{c}\right)<\lambda_{\mathbf{s}}$. Note that there exists $t>0$ such that $t\left(\phi_{1}, \cdots, \phi_{k}\right)+\left(\varphi_{1}, \cdots, \varphi_{k}\right) \geq 0$ in $\bar{\Omega}$. Let $\bar{t}=\inf \left\{t \in \mathbb{R}^{+} \mid t\left(\phi_{1}, \cdots, \phi_{k}\right)+\left(\varphi_{1}, \cdots, \varphi_{k}\right) \geq 0\right\}$. Apparently, $\bar{t}>0$ and $\bar{t} \phi_{i}+\varphi_{i} \geq 0$ in $\bar{\Omega}$ for all $i$. Notice that

$$
(-\Delta)^{s_{i}}\left(t \phi_{i}+\varphi_{i}\right)+\left[c_{i, i}-\lambda_{\mathbf{s}}\right]\left(t \phi_{i}+\varphi_{i}\right) \geq 0 \text { in } \Omega, \quad t \phi_{i}+\varphi_{i}=0 \text { in } \mathbb{R}^{n} \backslash \Omega .
$$

It follows from the same reasoning that either $\left(\bar{t} \phi_{1}+\varphi_{1}, \cdots, \bar{t} \phi_{k}+\varphi_{k}\right)>0$ in $\Omega$ or $\left(\bar{t} \phi_{1}+\right.$ $\left.\varphi_{1}, \cdots, \bar{t} \phi_{k}+\varphi_{k}\right)=0$ in $\Omega$. If the former is true, then Lemma 2.2 implies that $\bar{t} \phi_{i}(x)+\varphi_{i}(x) \geq$ $\beta_{i} d(x, \partial \Omega)^{s_{i}}$ in $\Omega$ for some $\beta_{i}>0$. Let $\beta=\min _{1 \leq i \leq k}\left\{\beta_{i}\right\}$. Since $\phi_{i}(x) \leq C d(x, \partial \Omega)^{s_{i}}$, there exists $t^{\prime} \in(0, \bar{t})$ for which $t^{\prime} \phi_{i}(x) \leq \frac{\beta}{2} d(x, \partial \Omega)^{s_{i}}$. Now let $\tau=\bar{t}-t^{\prime}$. It is clear that $\tau \in(0, \bar{t})$ and $\tau \phi_{i}+\varphi_{i} \geq \frac{\beta}{2} d(x, \partial \Omega)^{s_{i}}$ for all $x \in \Omega$, which contradicts the definition of $\bar{t}$. Consequently, $\left(\varphi_{1}, \cdots, \varphi_{k}\right)=-\bar{t}\left(\phi_{1}, \cdots, \phi_{k}\right)$. However, this also gives rise to a contradiction if $\mathfrak{s}\left(\mathcal{L}_{c}\right)<\lambda_{\mathbf{s}}$, which confirms that $\mathfrak{s}\left(\mathcal{L}_{c}\right)=\lambda_{\mathbf{s}}$. The above argument also shows that no other eigenvalue of $\mathcal{L}_{c}$ has a corresponding positive eigenfunction. In addition, the same argument leads to the fact that $\operatorname{ker}\left(\lambda_{\mathbf{s}} I-\mathcal{L}_{c}\right)=\operatorname{span}\left\{\left(\phi_{1}, \cdots, \phi_{k}\right)\right\}$. To show that $\operatorname{ker}\left(\lambda_{\mathbf{s}} I-\mathcal{L}_{c}\right)^{2}=\operatorname{ker}\left(\lambda_{\mathbf{s}} I-\mathcal{L}_{c}\right)$, we let $\mathcal{L}_{c}^{*}$ be the linear operator associated with $a^{*}(u, u)$, where $a^{*}(u, u): X \times X \rightarrow \mathbb{R}$ is given by

$$
a^{*}(u, v)=\sum_{j=1}^{k}\left\{\frac{c_{n, s_{j}}}{2} \int_{\mathbb{R}^{n}} \int_{\mathbb{R}^{n}} \frac{\left[u_{j}(x)-u_{j}(y)\right]\left[v_{j}(x)-v_{j}(y)\right]}{|x-y|^{n+2 s_{j}}} d y d x+\sum_{i=1}^{k} \int_{\Omega} c_{j, i} u_{i} v_{j} d x\right\} .
$$

Namely, $\mathcal{L}_{c}^{*}$ is the adjoint operator of $\mathcal{L}_{c}$. Hence $\lambda_{\mathbf{s}}$ is an eigenvalue of $\mathcal{L}_{c}^{*}$, and there exists a nonnegative eigenfunction $\left(\phi_{1}^{*}, \cdots, \phi_{k}^{*}\right)$ of $\mathcal{L}_{c}^{*}$ corresponding to $\lambda_{\mathbf{s}}$ with $\phi_{i}^{*} \in C^{s_{i}}\left(\mathbb{R}^{n}\right)$. The same argument given above implies that $\phi_{i}^{*}>0$ for each $i$. Thus, the Fredholm alternative yields that $\operatorname{ker}\left(\lambda_{\mathbf{s}} I-\mathcal{L}_{c}\right)^{2}=\operatorname{ker}\left(\lambda_{\mathbf{s}} I-\mathcal{L}_{c}\right)$. To conclude the proof, we let $\lambda^{\prime}=\inf _{u \in X,\|u\|_{X}=1} a(u, u)$ and show that $\lambda^{\prime}=\lambda_{\mathbf{s}}$. In light of [9], $\lambda^{\prime}$ is attained by some $w \in X$, and $\inf _{u \in X,\|u\|_{X}=1} a(u, u)$ is an eigenvalue of $\mathcal{L}_{c}$. Then, from the fact that $a(|w|,|w|) \leq a(w, w)$, we infer that $|w|$ is an eigenfunction associated with $\inf _{u \in X,\|u\|_{X}=1} a(u, u)$. Since $\lambda_{s}$ is the only eigenvalue of $\mathcal{L}_{c}$ with positive eigenfunctions, we must have $\lambda_{\mathbf{s}}=\inf _{u \in X,\|u\|_{X}=1} a(u, u)$. The proof is completed.

Thereafter, given $\mathbf{c}=\left\{c_{i, j}\right\}_{1 \leq i, j \leq k}$ with $c_{i, j} \in L^{\infty}(\Omega)$. Suppose that $\mathbf{c}=\left\{c_{i, j}\right\}_{1 \leq i, j \leq k}$ satisfies the condition given in Theorem 3.1, we shall write

$$
\left\{\begin{array}{l}
\left(\mathbf{L}_{\mathbf{c}} u\right)(x):=(-\Delta)^{s_{i}} u_{i}+\sum_{j=1}^{k} c_{i, j}(x) u_{j}, \quad x \in \Omega(1 \leq i \leq k) \\
\mathbf{D} u:=u, \quad x \in \mathbb{R}^{n} \backslash \Omega .
\end{array}\right.
$$

Let $\lambda_{\mathbf{s}}$ be given in Theorem 3.1, then $\lambda_{\mathbf{s}}$ is referred to as the principal eigenvalue of $\left\{\mathbf{L}_{\mathbf{c}}, \mathbf{D}, \Omega\right\}$. 
Definition 7. Let $u=\left(u_{1}, \cdots, u_{k}\right) \in C\left(\bar{\Omega}, \mathbb{R}^{k}\right) \cap L_{\text {loc }}^{\infty}\left(\mathbb{R}^{n}, \mathbb{R}^{k}\right)$, $u$ is said to be a classical supersolution of $\left\{\mathbf{L}_{\mathbf{c}}, \mathbf{D}, \Omega\right\}$ if $\mathbf{L}_{\mathbf{c}} u \in L^{\infty}\left(\Omega, \mathbb{R}^{k}\right),(-\Delta)^{s_{i}} u_{i}+\sum_{j=1}^{k} c_{i, j} u_{j} \geq 0$ in $\Omega$, and $u_{i} \geq 0$ in $\mathbb{R}^{n} \backslash \Omega$ for all $i \in\{1, \cdots, k\}$. In addition, $u$ is said to be a strict super-solution of $\left\{\mathbf{L}_{\mathbf{c}}, \mathbf{D}, \Omega\right\}$ provided that $u$ is a (classical) super-solution of $\left\{\mathbf{L}_{\mathbf{c}}, \mathbf{D}, \Omega\right\}$ while $u$ is not classical solution of $\mathbf{L}_{\mathbf{c}} u=0$ in $\Omega$ and $u=0$ in $\mathbb{R}^{n} \backslash \Omega$.

Definition 8. Let $\mathbf{L}_{\mathbf{c}}$ and $\mathbf{D}$ be defined in (3.4). Then $\left\{\mathbf{L}_{\mathbf{c}}, \mathbf{D}, \Omega\right\}$ is said to satisfy the strong maximum principle if $u_{i}>0$ in $\Omega$ for each $i \in\{1, \cdots, k\}$ whenever $u=\left(u_{1}, \cdots, u_{k}\right) \in$ $L_{\text {loc }}^{\infty}\left(\mathbb{R}^{n}, \mathbb{R}^{k}\right)$ with $u_{i} \in C^{s_{i}}(\bar{\Omega})$ for each $i$ is a nontrivial super-solution of $\left\{\mathbf{L}_{\mathbf{c}}, \mathbf{D}, \Omega\right\}$.

Theorem 3.2. Assume that $\Omega \subset \mathbb{R}^{n}$ is a bounded domain and is of class $\mathcal{C}^{1}$. Let $\left\{\mathbf{L}_{\mathbf{c}}, \mathbf{D}, \Omega\right\}$ be defined in (3.4). Assume that $\left\{c_{i, j}\right\}_{1 \leq i, j \leq k}$ satisfies the condition given in Theorem 3.1. Let $\lambda_{\mathbf{s}}$ be the principal eigenvalue of $\left\{\mathbf{L}_{\mathbf{c}}, \mathbf{D}, \Omega\right\}$. Then the following statements are equivalent

(i) $\lambda_{\mathbf{s}}>0$,

(ii) $\left\{\mathbf{L}_{\mathbf{c}}, \mathbf{D}, \Omega\right\}$ has a positive strict super-solution $u \in C\left(\bar{\Omega}, \mathbb{R}^{k}\right) \cap L_{\text {loc }}^{\infty}\left(\mathbb{R}^{n}, \mathbb{R}^{k}\right)$,

(iii) $\left\{\mathbf{L}_{\mathbf{c}}, \mathbf{D}, \Omega\right\}$ satisfies the strong maximum principle;

(iv) the linear boundary value problem

$$
\left\{\begin{array}{l}
(-\Delta)^{s_{i}} u_{i}+\sum_{j=1}^{k} c_{i, j}(x) u_{j}=f_{i} \quad \text { in } \Omega,(1 \leq i \leq k) \\
u_{i}=0 \text { in } \mathbb{R}^{n} \backslash \Omega
\end{array}\right.
$$

has an unique solution $u \in X$ for each $f=\left(f_{1}, \cdots, f_{k}\right)$ with $f_{i} \in L^{2}(\Omega)$. In particular, $u_{i} \geq 0$ for all $i$ provided that $f_{i} \geq 0$ for each $i \in\{1, \cdots, k\}$.

Proof. The proof is very similar to the one for Theorem 2.1. We just omit details.

Corollary 3.3. Let $\Omega_{i}(i=1,2) \subset \mathbb{R}^{n}$ be two bounded open domains with $\partial \Omega_{i} \in \mathcal{C}^{1}$ such that $\Omega_{2} \subseteq \Omega_{1}$. Denote the principal eigenvalues of $\left\{\mathbf{L}_{\mathbf{c}}, \mathbf{D}, \Omega_{i}\right\}$ by $\lambda_{\Omega_{i}}$, respectively. Then $\lambda_{\Omega_{1}} \leq \lambda_{\Omega_{2}}$. In case that $\Omega_{2} \subsetneq \Omega_{1}$, then $\lambda_{\Omega_{1}}<\lambda_{\Omega_{2}}$. Let $\lambda_{\mathbf{c}_{I}}$ be the principal eigenvalue of $\left\{\mathbf{L}_{\mathbf{c}_{I}}, \mathbf{D}, \Omega\right\}$ with $\mathbf{c}_{I}=\left\{c_{i, j}^{I}\right\}_{1 \leq i, j \leq k}$ and $c_{i, j}^{I} \in L^{\infty}(\Omega) ;(I=1,2)$. Assume that $c_{i, j}^{1} \leq c_{i, j}^{2}$. Then $\lambda_{\mathbf{c}_{1}} \leq \lambda_{\mathbf{c}_{2}}$. Moreover, if there exist $i$ and $j$ such that $c_{i, j}^{1} \lesseqgtr c_{i, j}^{2}$ in $\Omega$, then $\lambda_{\mathbf{c}_{1}}<\lambda_{\mathbf{c}_{2}}$.

Proof. In light of theorem 3.2, the proof is the same as the one for Proposition 2.5. The proof is completed.

Corollary 3.4. Suppose that all of the assumptions of Theorem 3.2 are satisfied. Let $u \in C\left(\mathbb{R}^{n}\right) \cap$ $C^{s}(\bar{\Omega})$ be a strict super-solution of $\left\{\mathbf{L}_{\mathbf{c}}, \mathbf{D}, \Omega\right\}$. Then exists a positive constant $\omega$, depending only on $n, s_{i}, c_{i, j}$, such that $u \geq 0$ whenever $|\Omega| \leq \omega$. 
Proof. Let $\lambda_{\mathbf{s}}$ be the principle eigenvalue of $\left\{\mathbf{L}_{\mathbf{c}}, \mathbf{D}, \Omega\right\}$. In view of Theorem 3.2, it is sufficient to show that $\lambda_{\mathbf{s}}>0$ if $|\Omega| \leq \omega$. Let $\phi=\left(\phi_{1}, \cdots, \phi_{k}\right)$ be an eigenfunction corresponding to $\lambda_{\mathbf{s}}$ with $\|\phi\|_{Y}=1$. By virtute of Proposition 2.4 and Theorem 3.1, we find that

$\lambda_{\mathbf{s}} \geq \sum_{i=1}^{k} \frac{c_{n, s_{i}}}{2} \int_{\mathbb{R}^{n}} \frac{\left|\phi_{i}(x)-\phi_{i}(y)\right|^{2}}{|x-y|^{n+2 s_{i}}} d y d x-2 \max _{1 \leq i, j \leq k}\left|c_{i, j}\right|_{L^{\infty}} \geq \sum_{i=1}^{k} \frac{K\left(n, s_{i}\right)}{r^{2 s_{i}}}-2 \max _{1 \leq i, j \leq k}\left|c_{i, j}\right|_{L^{\infty}}$,

where $r$ is the positive constant such that $\left|B_{r}(0)\right|=|\Omega|$, and $K\left(n, s_{i}\right)>0$ is a constant only depending on $n$ and $s_{i}$. Therefore, there exists $\omega>0$ such that $\lambda_{\mathbf{s}}>0$ if $0<r \leq \omega$. The proof is completed.

\section{Applications}

Proposition 4.1. Let $\Omega \subset \mathbb{R}^{n}$ be a bounded domain with $\partial \Omega \in \mathcal{C}^{1}$. Suppose that $u, v \in L_{\text {loc }}^{\infty}\left(\mathbb{R}^{n}\right) \cap$ $C^{s}(\bar{\Omega})$ satisfy

$$
\begin{aligned}
& (-\Delta)^{s} u-f(x, u) \geq 0 \geq(-\Delta)^{s} v-f(x, v), \quad x \in \Omega, \\
& u \gtrless 0 \text { in } \mathbb{R}^{n}, \text { and } u \geq v \text { in } \mathbb{R}^{n} \backslash \Omega .
\end{aligned}
$$

Here $f \in C^{0,1}(\bar{\Omega} \times \mathbb{R})$ satisfies that $f(x, 0)=0$ for any $x \in \bar{\Omega}$. Assume further that $t f(x, z) \geq$ $f(x, t z)$ in $\bar{\Omega}$ for any $t>1, z>0$, and there exist $x^{\prime} \in \Omega$ and $\bar{B}_{r}\left(x^{\prime}\right) \subset \Omega$ such that $t f(x, z)>$ $f(x, t z)$ for any $x \in \bar{B}_{r}(x)$, and any $t>1, z>0$, where $r>0$. Then $u \geq v$ in $\mathbb{R}^{n}$.

Proof. The argument is similar to the one adopted in the proof of Theorem 2.1. We shall give a sketch. Let $(u-v)\left(x_{0}\right)=\min _{x \in \bar{\Omega}}(u-v)(x)$. Again we need to show that $(u-v)\left(x_{0}\right) \geq 0$. Note that $(u-v)(x) \geq 0$ for all $x \in \mathbb{R}^{n} \backslash \Omega$ and $u-v \in C^{s}(\bar{\Omega})$. Hence, there exists $\kappa>0$ for which $(u-v)(x) \geq-\kappa d(x, \partial \Omega)^{s}$ for all $x \in \Omega$. On the other hand, the assumptions show that $(-\Delta)^{s} u+\frac{f(x, u)}{u} u \geq 0$ in $\Omega$ and $u \gtrless 0$. Therefore, Lemma 2.2 implies that there is $\beta>0$ for which $u \geq \beta d(x, \partial \Omega)^{s}$ for all $x \in \Omega$. Now assume that the desired conclusion is not true, that is, $(u-v)\left(x_{0}\right)<0$. Then, as the proof of Theorem 2.1, let $\bar{t}=\inf \{t \in \mathbb{R} \mid(t+1) u(x)-v(x) \geq$ 0 in $\bar{\Omega}\}$. Clearly, $\bar{t}>0$. Write $w=(\bar{t}+1) u-v$. Due the assumptions made on $f$, there holds

$$
(-\Delta)^{s}(\bar{t}+1) u-f(x,(\bar{t}+1) u) \gtrless 0, \quad(-\Delta)^{s} w-\frac{f(x,(\bar{t}+1) u)-f(x, v)}{(\bar{t}+1) u-v} w \gtrless 0, \quad x \in \Omega .
$$

Consequently, there exists $\gamma>0$ such that $(\bar{t}+1) u-v \geq \gamma d(x, \partial \Omega)^{s}$ for all $x \in \bar{\Omega}$. Hence the rest proof follows from the same reasoning given in the proof of Theorem 2.1. The proof is completed. 
Theorem 4.1. Suppose that all the assumptions of Proposition 4.1 on $\Omega$ and $f$ are satisfied. Assume further that $f \in C^{0,1+\alpha}(\bar{\Omega} \times \mathbb{R})$ for some $\alpha \in(0,1)$, and there exists $M>0$ for which $f(x, u) \leq 0$ for all $x \in \Omega$ whenever $u \geq M$. Let $\lambda_{s}$ be the principle eigenvalue of $\left\{L_{c}, \mathcal{D}, \Omega\right\}$, where $c(x)=$ $-f_{z}(x, 0)$. Then the following boundary value problem

$$
\left\{\begin{array}{l}
(-\Delta)^{s} u=f(x, u), \quad x \in \Omega \\
u=0, \quad x \in \mathbb{R}^{n} \backslash \Omega
\end{array}\right.
$$

has an unique nontrivial bounded positive solution if and only if $\lambda_{s}<0$. Here $0<s<1$.

Proof. We first prove the only if part by an old trick. Let $u \in L^{\infty}(\Omega)$ be the positive solution, and let $\varphi$ be a positive eigenfunction associated with $\lambda_{s}$. Now multiplying the both sides of (4.1) by $\varphi$ and integrating resulting equation over $\Omega$ yield that

$$
\begin{aligned}
0 & =\int_{\Omega}\left[(-\Delta)^{s} u \varphi-f(x, u) \varphi\right] d x \\
& =\int_{\Omega}\left[u(-\Delta)^{s} \varphi-f(x, u) \varphi\right] d x \\
& =\int_{\Omega}\left\{\lambda_{s}+\left[f_{z}(x, 0)-\frac{f(x, u)}{u}\right]\right\} u \varphi d x .
\end{aligned}
$$

Notice that $u, \varphi>0$ in $\Omega$, and $f_{z}(x, 0) \geq f(x, u) / u$ in $\Omega$. In particular, the assumption implies that there exist a $x^{\prime} \in \Omega$ and an open neighborhood $B_{r^{\prime}}\left(x^{\prime}\right)$ of $x^{\prime}$ such that $f_{z}(x, 0)>f(x, u) / u$ for all $x \in B_{r^{\prime}}\left(x^{\prime}\right)$. Consequently, $\lambda_{s}<0$. We now proceed to show that (4.1) has a bounded positive solution provided that $\lambda_{s}<0$. Since $f(x, t \varphi)-t f_{z}(x, 0) \varphi=\left(\int_{0}^{1} f_{z}(x, \tau t \varphi) d \tau-\right.$ $\left.f_{z}(x, 0)\right) t \varphi$, there exists $t_{1}>0$, which is sufficiently small such that $f\left(x, t_{1} \varphi\right) \geq t_{1} f_{z}(x, 0) \varphi+$ $\frac{\lambda_{s}}{2} t_{1} \varphi$ and $t_{1} \varphi<M$. Thus, $(-\Delta)^{s} t_{1} \varphi-f\left(x, t_{1} \varphi\right) \leq 0$ in $\Omega$ and $t_{1} \varphi=0$ in $\mathbb{R}^{n} \backslash \Omega$.

Now set $X=\left\{u \in C\left(\mathbb{R}^{n}\right) \mid u=0\right.$ in $\left.\mathbb{R}^{n} \backslash \Omega\right\}$, and $Y=\{u \in C(\bar{\Omega}) \mid u=0$ on $\partial \Omega\}$. Let $J: X \rightarrow Y$ be given by $J u:=\left.u\right|_{\bar{\Omega}}$. Clearly, $J$ is a linear bijection. Given $h \in Y$, we let $\left.u=(-\Delta)^{s}+\rho I\right)^{-1} h$ be the unique (weak) solution of

$$
\left\{\begin{array}{l}
(-\Delta)^{s} u+\rho u=h, \quad x \in \Omega, \\
u=0, \quad x \in \mathbb{R}^{n} \backslash \Omega .
\end{array}\right.
$$

Here $\rho>0$ is a constant. In view of Proposition 1.1 of [21], $\left.(-\Delta)^{s}+\rho I\right)^{-1} h \in C^{s}\left(\mathbb{R}^{n}\right)$ is also a classic solution of (4.2). Next we let $\mathcal{F}: X \rightarrow X$ be defined by $\left.\mathcal{F} u:=(-\Delta)^{s}+\rho I\right)^{-1} F(x, J u)$, where $F(x, z)=\rho z+f(x, z)$. We now show that $\mathcal{F}$ is continuous and compact. In fact, given that $h_{1}, h_{2} \in X$, assume without loss of generality that $\left\|h_{1}-h_{2}\right\|_{X} \leq 1$, and $\left\|h_{1}\right\| \leq M$, it follows from Theorem 2.1 that

$$
\left\|\mathcal{F} h_{1}-\mathcal{F} h_{2}\right\|_{L^{2}(\Omega)} \leq C_{\rho}\left\|F\left(x, J h_{1}\right)-F\left(x, J h_{2}\right)\right\|_{L^{2}(\Omega)} \leq C(\rho, f, M)\left\|h_{1}-h_{2}\right\|_{X} .
$$


Note that $(-\Delta)^{s}\left(\mathcal{F} h_{1}-\mathcal{F} h_{2}\right)+\rho\left(\mathcal{F} h_{1}-\mathcal{F} h_{2}\right)=F\left(x, h_{1}\right)-F\left(x, h_{2}\right)$. It follows from Proposition 2.1 and Proposition 1.1 of [21] that

$$
\left\|\mathcal{F} h_{1}-\mathcal{F} h_{2}\right\|_{C^{s}\left(\mathbb{R}^{n}\right)} \leq C^{\prime}(\rho, f, M, \Omega)\left\|h_{1}-h_{2}\right\|_{X}
$$

Therefore, $\mathcal{F}$ is continuous. In case that $h_{2}=0$, for any $h \in X$ with $\|h\|_{X} \leq M$, It is clear that $\|\mathcal{F} h\|_{C^{s}\left(\mathbb{R}^{n}\right)} \leq C^{\prime}(\rho, f, M, \Omega) M$. Hence Ascoli-Arzelà theorem implies that $\mathcal{F}$ is also compact. Next we let $X_{\varphi, M}:=\left\{u \in X \mid t_{1} \varphi \leq u \leq M\right\}$. Apparently, $X_{\varphi, M}$ is a close and convex subspace of $X$. Let $\rho$ be sufficiently large such that $\rho>L(M)$, where $L(M)$ is the Lipschitz constant for $f(x, z)$ over $z \in[0, M]$. Then $F(x, z)$ is non-decreasing in $z$. Thanks to the fact that

$$
(-\Delta)^{s} t_{1} \varphi+\rho\left(t_{1} \varphi\right) \leq F\left(x, t_{1} \varphi\right), x \in \Omega, \quad(-\Delta)^{s} M+\rho M \geq F(x, M), x \in \Omega,
$$

from Theorem 2.1, we infer that $t_{1} \varphi \leq \mathcal{F} h \leq M$ as long as $h \in X_{\varphi, M}$. Thus, it follows from Schauder's fixed point theorem that $\mathcal{F}$ has a fixed point in $X_{\varphi, M}$. Namely, (4.1) has a solution in $X_{\varphi, M}$. We now proceed to show the uniqueness of the bounded positive solution of (4.1). Let $u_{1}$ and $u_{1}$ be two bounded positive classic solutions of (4.1), in view of Proposition 4.1, by interchanging the role of $u_{1}$ and $u_{2}$ as a super-solution and a sub-solution, we find that $u_{1}=u_{2}$. The proof is completed.

Theorem 4.2. Let $\Omega \subset \mathbb{R}^{n}$ be a bounded domain with $\partial \Omega \in \mathcal{C}^{1}$. Let $f=\left(f_{1}, \cdots, f_{k}\right) \in$ $C^{0,2+\alpha}\left(\bar{\Omega} \times \mathbb{R}^{k}, \mathbb{R}^{k}\right)$ satisfies that $f(x, 0)=0$ for all $x \in \bar{\Omega}$. Given $z=\left(z_{1}, \cdots, z_{k}\right)$, let $c_{i, j}(x, z)=-\partial_{j} f_{i}$, where $\partial_{j} f_{i}(x, z)$ stands for the partial derivative of $f_{i}$ with respect to $z_{j}$. Suppose that there exist $k$ positive constants $M_{i}(1 \leq i \leq k)$ such that $c_{i, j}(x, z) \leq 0$ for any $(x, z) \in \bar{\Omega} \times \prod_{i=1}^{k}\left[0, M_{i}\right]$ and $i \neq j$. Moreover, there exist a permutation $p_{1} p_{2} \cdots p_{k}$ of $\{1, \cdots, k\}$ and subsets $\Omega_{p_{i} p_{i+1}}(1 \leq i \leq k-1)$ and $\Omega_{p_{k} p_{1}}$ such that $c_{p_{i} p_{i+1}}<0$ for any $(x, z) \in$ $\Omega_{p_{i} p_{i+1}} \times \prod_{i=1}^{k}\left[0, M_{i}\right)$, and $c_{p_{k} p_{1}}<0$ for any $(x, z) \in \Omega_{p_{k} p_{1}} \times \prod_{i=1}^{k}\left[0, M_{i}\right)$. In particular, $c_{i, j}(x, 0) \leq c_{i, j}(x, z)$ for all $(x, z) \in \bar{\Omega} \times \prod_{i=1}^{k}\left[0, M_{i}\right]$, and there exist some $i, j$ and $x_{1} \in \Omega$ such that $c_{i, j}(x, 0)<c_{i, j}(x, z)$ for any $x \in \bar{B}_{r_{1}}\left(x_{1}\right) \times \prod_{i=1}^{k}\left(0, M_{i}\right]$, where $r_{1}>0$ and $B_{r_{1}}\left(x_{2}\right) \subset \Omega$. Assume further that for each $i, f_{i} \leq 0$ whenever $\left(z_{1}, \cdots, z_{i}, \cdots, z_{k}\right) \geq\left(0, \cdots, M_{i}, \cdots 0\right)$. Let $0<s_{i}<1(1 \leq i \leq k)$. Suppose that $2 \min _{1 \leq i \leq k} s_{i} \geq \max _{1 \leq i \leq k} s_{i}$. Then the following boundary value problem

$$
\left\{\begin{array}{l}
(-\Delta)^{s_{i}} u_{i}=f_{i}(x, u), \quad x \in \Omega \\
u_{i}=0, \quad x \in \mathbb{R}^{n} \backslash \Omega
\end{array}\right.
$$

has a bounded positive solution $u$ with $u=\left(u_{1}, \cdots, u_{k}\right)$ and $u_{i} \in\left[0, M_{i}\right]$ if and only if $\lambda_{\mathbf{s}}<0$. Here $\lambda_{\mathbf{s}}$ is the principal eigenvalue of $\left\{\mathbf{L}_{\mathbf{c}}, \mathcal{D}, \Omega\right\}$ with $\mathbf{c}=\left\{c_{i, j}(x, 0)\right\}_{1 \leq i, j \leq k}$. 
Proof. We first prove the non-existence of a bounded positive solution $u$ with $u_{i} \in\left[0, M_{i}\right]$ provided that $\lambda_{\mathbf{s}} \geq 0$. Assume to the contrary that (4.3) has a bounded positive solution $u$ with $u_{i} \in\left[0, M_{i}\right]$ when $\lambda_{\mathbf{s}} \geq 0$. Then Proposition 1.1 of [21] implies that $u_{i} \in C^{s_{i}}\left(\mathbb{R}^{n}\right)$. Let $x^{i} \in \Omega$ be a point such that $u_{i}\left(x^{i}\right)=\max _{x \in \mathbb{R}^{n}} u_{i}(x)$, then we see that $0<(-\Delta)^{s_{i}} u_{i}\left(x^{i}\right)=f_{i}\left(x^{i}, u\left(x^{i}\right)\right)$ unless $u_{i}(x)=0$ for all $x \in \mathbb{R}^{n}$. Thus, the assumption implies that $0 \leq u_{i}(x) \leq u_{i}\left(x^{i}\right)<M_{i}$ for each $i$. In addition, since $f(x, 0)=0$, we have

$$
\widetilde{c}_{i, j}(x):=-\int_{0}^{1} \partial_{j} f_{i}\left(x, t u_{1}(x), \cdots, t u_{k}(x)\right) d t, \quad(-\Delta)^{s_{i}} u_{i}+\sum_{j=1}^{k} \widetilde{c}_{i, j}(x) u_{j}=0, \quad x \in \Omega .
$$

Hence the assumption shows that $u_{i}(x)>0$ in $\Omega$ for each $i$, and consequently, 0 is the principal eigenvalue of $\left\{\mathbf{L}_{\widetilde{\mathbf{c}}}, \mathbf{D}, \Omega\right\}$. Here $\widetilde{\mathbf{c}}=\left\{\widetilde{c}_{i, j}\right\}_{1 \leq i, j \leq k}$. Due to the assumption, $\widetilde{c}_{i, j}(x) \geq c_{i, j}(x, 0)$ for $1 \leq i, j \leq k$. Moreover, it is easy to see that

$$
\widetilde{c}_{i, j}(x)-c_{i, j}(x, 0) \geq \int_{\frac{1}{2}}^{1}\left[\partial_{j} f_{i}(x, 0)-\partial_{j} f_{i}\left(x, t u_{1}(x), \cdots, t u_{k}(x)\right)\right] d t .
$$

In view of the assumption, there exist some $i, j$ such that $\widetilde{c}_{i, j}(x) \gtrless c_{i, j}(x, 0)$ in $\Omega$. It then follows from Corollary 3.3 that $\lambda_{\mathbf{s}}<0$, which is a contradiction. We now proceed to show the existence of a bounded positive solution to (4.3) given that $\lambda_{\mathbf{s}}<0$. The argument is almost the same as the one for Theorem 4.1. Only a sketch is needed. Let $\varphi=\left(\varphi_{1}, \cdots, \varphi_{k}\right)$ be a positive eigenfunction for $\lambda_{\mathbf{s}}$ with $|\varphi|_{L^{\infty}}=1$. Note that $(-\Delta)^{s_{i}} \varphi_{i}+c_{i i}(x, 0) \varphi_{i} \geq 0$ in $\Omega$, it follows from Lemma 2.2 that $\varphi_{i} \geq \beta_{i} d^{s_{i}}(x, \partial \Omega)$ for some $\beta_{i}>0$, where $d^{s_{i}}(x)=d^{s_{i}}(x, \partial \Omega)$. Meanwhile, since $\varphi_{i} \in C^{s_{i}}\left(\mathbb{R}^{n}\right)$ and $\varphi_{i}=0$ in $\Omega^{c}$. there exists $C_{i}>0$ for which $\varphi_{i} \leq C_{i} d^{s_{i}}(x)$. Given $t \in(0,1)$, for each $x \in \Omega$, applying Taylor冈s formula gives

$$
\begin{aligned}
\left|f_{i}(x, t \varphi)-\sum_{j=1}^{k} \partial_{j} f_{i}(x, 0) t \varphi_{j}\right| & =\left|\sum_{\tau, j=1}^{k} t^{2} \varphi_{j} \varphi_{\tau} \int_{0}^{1}\left[(1-\theta) \partial_{j} \partial_{\tau} f_{i}(x, \theta t \varphi)\right] d \theta\right| \\
& \leq \sum_{\tau, j=1}^{k}\left[\eta_{i} t^{2} \varphi_{j} \varphi_{\tau}\right]
\end{aligned}
$$

where $\eta_{i}=\max _{1 \leq \tau, j \leq k}\left\{\sup _{(x, z) \in \bar{\Omega} \times[0,1]^{k}}\left|\partial_{\tau} \partial_{j} f_{i}\right|\right\}$. In view of the assumption, we may assume without loss of generality that $0<s_{1} \leq s_{2} \cdots \leq s_{i} \leq \cdots \leq s_{k}$, so $2 s_{1} \geq s_{k}$, and we have

$$
0<\eta_{i} \frac{\sum_{\tau, j=1}^{k} t^{2} \varphi_{\tau} \varphi_{j}}{t \varphi_{i}} \leq t \eta_{i} \frac{\sum_{\tau, j=1}^{k} C_{\tau} C_{j} d^{s_{\tau}+s_{j}}(x)}{\beta_{i} d^{s_{i}}(x)} .
$$

Since for any $i, j, \tau \in\{1, \cdots, k\}, s_{\tau}+s_{j} \geq 2 s_{1} \geq s_{k} \geq s_{i}$, it follows that

$$
\lim _{t \rightarrow 0} t \eta_{i} \frac{\sum_{\tau, j=1}^{k} C_{\tau} C_{j} d^{s_{\tau}+s_{j}}(x)}{\beta_{i} d^{s_{i}}(x)}=0 \quad \text { uniformly in } \Omega .
$$


Thus, there exists $t_{1}>0$ sufficiently small such that $f_{i}\left(x, t_{1} \varphi\right) \geq t_{1} \sum_{j=1}^{k} \partial_{j} f_{i}(x, 0) \varphi_{j}+t_{1} \frac{\lambda_{s}}{2} \varphi_{i}$ in $\Omega$, and $t_{1} \varphi_{i}<M_{i}$ for each $i$. Namely, $(-\Delta)^{s_{i}} t_{1} \varphi_{i}-f_{i}\left(x, t_{1} \varphi\right) \leq 0$ in $\Omega$ for each $i$. On the other hand, we have $(-\Delta)^{s_{i}} M_{i}-f_{i}\left(x, M_{1}, \cdots, M_{k}\right) \geq 0$ in $\Omega$ for each $i$. Now let $\mathbf{F}$ : $\prod_{i=1}^{k} X_{i} \rightarrow \prod_{i=1}^{k} X_{i}$ be defined by

$$
\begin{aligned}
\left.\mathbf{F} u:=\left((-\Delta)^{s_{1}}+\rho I\right)^{-1} F_{1}(x, J u), \cdots,(-\Delta)^{s_{i}}+\rho I\right)^{-1} F_{i}(x, J u), \\
\left.\left.\cdots,(-\Delta)^{s_{k}}+\rho I\right)^{-1} F_{k}(x, J u)\right),
\end{aligned}
$$

where $X_{i}=X$, which is specified in the proof of Theorem 4.1, and $J$ is the linear bijection given in the proof of Theorem 4.1. In addition, $F_{i}(x, z)=\rho z_{i}+f_{i}(x, z)$, and

$$
\rho \geq \max _{1 \leq i \leq k} \sup _{(x, z) \in \bar{\Omega} \times \prod_{i=1}^{k}\left[0, M_{i}\right]}\left|\partial_{i} f_{i}\right| .
$$

Clearly, $F_{i}(x, z)$ is nondecreasing in $z$. The same argument as that given in the proof of Theorem 4.1 yields that $\mathbf{F}$ is continuous and compact. Moreover, $t_{1} \varphi \leq \mathbf{F} h \leq M$ as long as $h \in \Xi_{\varphi, M}$, where $\Xi_{\varphi, M}=\left\{u \in \prod_{i=1}^{k} X_{i} \mid t_{1} \varphi_{1} \leq u_{i} \leq M_{i}, 1 \leq i \leq k\right\}$, and $M=\left(M_{1}, \cdots, M_{k}\right)$. Thus, Schauder's fixed point theorem shows that (4.3) has a solution $u$ with $0 \leq u_{i}<M_{i}$. The proof is completed.

The assumption of Theorem 4.2 on $s_{i}$ seems to be a bit restrictive, and could be relaxed via degree theory approach and should be dealt with in the near future. Nonetheless, it always holds if $s_{i} \in\left[\frac{1}{2}, 1\right)$ for all $i$. Theorem 4.2 may be used to study the existence and non-existence of non-negative equilibria of a nonlocal Ross-Macdonald model as follows:

$$
\left\{\begin{array}{l}
w_{t}+(-\Delta)^{s_{1}} w=a(x) b(x) m(x)(1-w) v-r_{1}(x) w, \quad(t, x) \in \mathbb{R}^{+} \times \Omega, \\
v_{t}+(-\Delta)^{s_{2}} v=a(x) c(x)(1-v) w-r_{2}(x) v, \quad(t, x) \in \mathbb{R}^{+} \times \Omega, \\
w=v=0, \quad(t, x) \in \mathbb{R}^{+} \times \Omega^{c} .
\end{array}\right.
$$

Here $a \gtrless 0, b \gtrless 0, c \gtrless 0 ; m \gtrless 0, r_{1} \gtrless 0$, and $r_{2} \gtrless 0$ in $\Omega$. The biological meanings of these parameters can be found in [25], $w$ and $v$ are the density of infected vectors and the density of infectious mosquitoes at $(t, x)$, respectively. Suppose that $0<s_{i}<1(i=1,2)$, and either $2 s_{1} \geq s_{2}, s_{1} \leq s_{2}$ or $2 s_{2} \geq s_{1}, s_{2} \leq s_{1}$. Then all of the assumptions of Theorem 4.2 on $f$ and $s_{i}$ are satisfied. Let $\lambda_{\mathbf{s}}$ be the principal eigenvalue of $\left\{\mathbf{L}_{\mathbf{c}}, \mathbf{D}, \Omega\right\}$, where $\mathbf{L}_{\mathbf{c}}$ is given by

$$
\mathbf{L}_{\mathbf{c}}(w, v)=\left((-\Delta)^{s_{1}} w+r_{1} w-a b m v,(-\Delta)^{s_{2}} v+r_{2} v-a c w\right) .
$$

Then Theorem 4.2 suggests that $\lambda_{\mathbf{s}}$ plays a role analogous to that of basic reproduction number, a crucial threshold in the Ross-Macdonald theory of mosquito-borne pathogen transmission. Thanks to Corollary $3.4, \lambda_{\mathbf{s}}>0$ if $|\Omega|$ is sufficiently small. Hence, a plausible biological implication of Theorem 4.2 is that spatial environments of small size can not sustain disease spreading since 0 is the only non-negative equilibrium in $[0,1] \times[0,1]$, which is linearly stable. 


\section{References}

[1] H. Amann and J. López-Gómez, A priori bounds and multiple solutions for superlinear indefinite elliptic problems, J. Differential Equations. 146 (1998), 336-374.

[2] H. Berestycki, L. Nirenberg, S. R. S. Varadhan, The principal eigenvalue and maximum principle for second-order elliptic operators in general domains, Comm. Pure Appl. Math., 47 (1994), 47-92.

[3] P. W. Bates and G. Zhao, Existence, uniqueness and stability of the stationary solution to a nonlocal evoluation equation arising in population dispersal, J. Math. Anal. Appl., 332 (2007), 428-440.

[4] P. W. Bates and G. Zhao, Spectral convergence and Turing Patterns for nonlocal diffusion systems, Preprint.

[5] L. Brasco, E. Lindgren, E. Parini, The fractional cheeger problem, Interfaces Free Bound., 16 (2014), 419-458.

[6] L. Brasco and E. Parini, The second eigenvalue of the fractional p-Laplacian, Adv. Calc. Var., 9 (2015), 323-355.

[7] L. Caffarelli, S. Dipierro, E. Valdinoci, A logistic equation with nonlocal interactions, Kinet. Relat. Models, 10 (2017), 141-170.

[8] R.S. Cantrell and C. Cosner, Diffusive logistic equations with indefinite weights: population models in a disrupted environments, Proc. Roy. Soc., Edinburgh, 112A (1989), 293-318.

[9] J. Chabrowski, Varational methods for potential operator equations, Walter de Gruyter, New York, 1997.

[10] J. Coville, On a simple criterion for the existence of a principal eigenfunction of some nonlocal operators, J. Differential Equations, 249 (2010), 2921-2953.

[11] Y. Du, Order structure and topological methods in nonlinear PDEs, Vol. 1. Maximum principles and applications, World Scientific, 2006.

[12] B. Dyda, Fractional calculus for power functions and eigenvalues of the fractional Laplacian, Fract. Calc. Appl. Anal., 15 (2012), 536-555.

[13] L. C. Evans, Partial Differential Equations, Amer. Math. Soc., Providence, RI, 1998.

[14] A. Fiscella, R. Servadei, E. Valdinoci, Density properties for fractional Sobolev spaces, Ann. Acad. Sci. Fenn. Math., 40 (2015), 235-253. 
[15] A. Greco and R. Servadei, Hopf's lemma and contrained radial symmetry for fractional Laplacian, Math. Res. Lett., 23 (2016), 863-885.

[16] B. Helffer, Spectral theory and its applications, Cambridge University Press, Cambridge, 2013.

[17] J. López-Gómez, Linear second order elliptic operators, World Scientific, 2013.

[18] J. López-Gómez and M. Molina-Meyer, The maximum principle for cooperative weakly elliptic systems and some applications, Diff. Int. Eqs., 7 (1994), 383-398.

[19] A. Massaccesi and E. Valdinoci, Is a nonlocal diffusion strategy convenient for biological populations in competition?, J. Math. Biol., 74(2017), 113-47.

[20] E. Di Nezza, G. Palatucci, E. Valdinoci, Hitchhiker's guide to the fractional Sobolev spaces, Bull. Sci. Math., 136 (2012), 521-573.

[21] X. Ros-Oton and J. Serra, The Dirchlet problem for the fractional Laplacian: regularity up to the boundary, J. Math. Pures. Appl., 101 (2014), 275-302.

[22] X. Ros-Oton and J. Serra, The Pohozaev identity for the fractional Laplacian, Arch. Rational Mech. Anal., 213 (2014), 587-628.

[23] R. Servadei and E. Valdinoci, Varational methods for non-local operators of elliptic type, Discrete Contin. Dyn. Syst., 33 (2013), 2105-2137.

[24] R. Servadei and E. Valdinoci, Lewy-Stampacchia type estimates for variational inequalities driven by (non)local operators, Rev. Mat. Iberoam., 29 (2013), 1091-1126.

[25] David L. Smith, Katherine E. Battle, Simon I. Hay, Christopher M. Barker, Thomas W. Scott and F. Ellis McKenzie, Ross, Macdonald, and a Theory for the Dynamics and Control of Mosquito-Transmitted Pathogens, PLoS Pathogens, 8 (2012), 1-13.

[26] G. Zhao and S. Ruan, Spatial and temporal dynamics of a nonlocal viral infection model, SIAM. J. Appl. Math., 78 (2018), 1954-1980.

Guangyu Zhao Department of Mathematics and Statistics, Auburn University, Auburn, AL 36849

E-mail: gzz0021@auburn.edu 